PIERRE DA SILVA PAZ JUNIOR

CONCORDÂNCIA INTEROBSERVADOR DOS ACHADOS DO

ELETROENCEFALOGRAMA EM PACIENTES NA FASE AGUDA E SUBAGUDA DO ACIDENTE CEREBROVASCULAR ISQUÊMICO

BRASÍLIA

2016 
UNIVERSIDADE DE BRASÍLIA

FACULDADE DE MEDICINA

PROGRAMA DE PÓS-GRADUAÇÃO EM CIÊNCIAS MÉDICAS

PIERRE DA SILVA PAZ JUNIOR

CONCORDÂNCIA INTEROBSERVADOR DOS ACHADOS DO

ELETROENCEFALOGRAMA EM PACIENTES NA FASE AGUDA E SUBAGUDA DO ACIDENTE CEREBROVASCULAR ISQUÊMICO

Dissertação apresentada como requisito parcial para a obtenção do Título de Mestre em Ciências Médicas pelo Programa de Pós-Graduação em Ciências Médicas da Universidade de Brasília.

Orientador: Professora Doutora Iruena Moraes Kessler Co-orientador: Doutor Wagner Afonso Teixeira.

BRASÍLIA

2016 
Ficha catalográfica elaborada automaticamente, com os dados fornecidos pelo(a) autor(a)

d

da Silva Paz Junior, Pierre

/ Pierre da Silva Paz Junior; orientador Iruena Moraes Kessler; co-orientador Wagner Afonso Teixeira. -- Brasilia, 2016.

65 p.

Tese (Doutorado - Mestrado em Ciências Médicas) Universidade de Brasilia, 2016.

1. eletroencefalograma. 2. acidente vascular cerebral isquêmico. 3. concordância interobservador. 4. EEG. I. Moraes Kessler, Iruena, orient. II. Afonso Teixeira, Wagner, co-orient. III. Título. 


\section{CONCORDÂNCIA INTER-OBSERVADOR DOS ACHADOS DO ELETROENCEFALOGRAMA EM PACIENTES NA FASE AGUDA E SUBAGUDA DO ACIDENTE CEREBROVASCULAR ISQUÊMICO}

Dissertação apresentada como requisito parcial para a obtenção do Título de Mestre em Ciências Médicas pelo Programa de Pós-Graduação em Ciências Médicas da Universidade de Brasília.

Aprovado em 1

BANCA EXAMINADORA

Professora Doutora Iruena Moraes Kessler Presidente da Banca - Universidade de Brasília

Professor Doutor Luiz Augusto Nascimento Membro Efetivo - Universidade de Brasília

Professor Doutor André Luiz Lopes Sampaio Membro Efetivo - Universidade de Brasília 
Membro Suplente - Universidade de Brasília 
À Thayana, minha esposa, por todo o amor, incentivo e paciência. Ao meu tio Leonardo, pelo auxílio, pelas longas horas de coorientação, incentivo e generosidade. Aos meus pais, Pierre e Suelene, que são as bases sólidas que estruturam minha caminhada pela vida. À minha orientadora pela compreensão, ajuda e solicitude. Aos professores, às técnicas em eletroencefalografia, aos colegas neurologistas e aos residentes de Neurologia, Eletroencefalografia e Neurocirurgia do Hospital de Base do Distrito Federal pela amizade e empenho ao sonhar e realizar juntos a missão de produzir conhecimento em um tempo de grandes adversidades. Aos pacientes e seus familiares que participaram da pesquisa pela confiança depositada, mesmo em um momento de tamanha dificuldade em suas vidas. 


\section{RESUMO}

OBJETIVO: Determinar os achados eletroencefalográficos e avaliar a taxa de concordância interobservador de pacientes com acidente vascular cerebral isquêmico durante a fase aguda atendidos em um hospital terciário do Distrito Federal. MATERIAIS E MÉTODOS: Foram realizados eletroencefalogramas (EEG) em pacientes com diagnóstico clínico e radiológico de acidente vascular cerebral isquêmico (AVC) avaliados entre 2014 a 2015 e realizada análise estatística pelo teste kappa para avaliação da taxa de concordância interobservador. RESULTADOS: Foram avaliados 18 pacientes com diagnóstico de AVC de ambos os sexos, com 21 a 77 anos, com média de 50 anos, tendo sido realizada trombólise endovenosa com alteplase em cerca de um terço destes. Todos os apresentaram confirmação radiológica das lesões. O EEG foi laudado como anormal em $78 \%$ dos pacientes. A atividade lenta focal (delta ou teta) foi o achado anormal mais frequente, sendo intermitente em cerca de $33 \%$ da amostra e contínua em $28 \%$ destes. Achados lateralizatórios e atividade epileptiforme foram encontrados em $61 \%$ e $11 \%$ dos casos, respectivamente. As taxas de concordância interexaminador encontradas foram substanciais em relação à simetria da atividade elétrica cerebral, à presença de atividade lenta anormal, à presença de atividade epileptiforme e à conclusão do EEG (normal versus anormal). A avaliação da organização da atividade de base e da lateralização dos achados anormais pelo EEG apresentaram concordância apenas moderada. CONCLUSÃO: A neuroimagem por tomografia e ressonância magnética de crânio continuam a ser os métodos diagnósticos mais empregados no atendimento do paciente com AVC em fase aguda a subaguda. $O$ EEG pode trazer informações sobre o gravidade e prognóstico clínico, embora apresente um grau de concordância interexaminador moderado a substancial. É necessária a implementação de medidas para melhorar o grau de concordância interexaminador dos exames de EEG, sobretudo através da publicação de terminologia simples, padronizada internacionalmente e do treinamento interativo dos eletroencefalografistas.

Palavras-chave: Eletroencefalografia, EEG, Acidente Vascular Cerebral, Concordância interexaminador. 


\begin{abstract}
OBJECTIVES: To determine the electroencephalographic findings and assess interrater concordance rate of patients with ischemic stroke during the acute phase attending a tertiary hospital in the Federal District. MATERIALS AND METHODS: Electroencephalogram (EEG) were performed in patients with clinical and radiological diagnosis of ischemic stroke (IS) evaluated between 2014-2015 and performed statistical analysis using the kappa test to evaluate the interobserver agreement rate. RESULTS: We evaluated 18 patients with stroke of both genders, 21-77 years, mean 50 years, having been held intravenous thrombolysis with alteplase in about a third of these. All presented radiologic confirmation of the lesions. The EEG was reported as abnormal in $78 \%$ of patients. The focal slow activity (delta and theta) was the most common abnormal finding, being intermittent about $33 \%$ of the sample and continuous in $28 \%$ thereof. Unilateral and epileptiform findings were found in $61 \%$ and $11 \%$ of cases, respectively. The interrater agreement rates were substantial in relation to the symmetry of brain electrical activity, the presence of abnormally slow activity, the presence of epileptiform activity and the conclusion of the EEG (normal versus abnormal). The evaluation of the organization's basic activity and lateralization of abnormal findings by EEG showed only moderate agreement. CONCLUSION: Neuroimaging by CT and skull MRI remains the diagnostic methods most used in patient care with stroke in acute or subacute phases. The EEG can provide information on the severity and clinical prognosis, but with a level of interrater agreement moderate to substantial. the implementation of measures is needed to improve the degree of interrater agreement of EEG tests, especially through the simple terminology of publication, internationally standardized and interactive training EEG readers.
\end{abstract}

Key-words: Electroencephalography, EEG, stroke, Interobserver agreement, interratter agreement. 


\section{Lista de Tabelas}

Tabela 1 - Características clínicas e demográficas da amostra 15

Tabela 2 - Características eletroencefalográficas da amostra. 17

Tabela 3 - Comparativo da atividade eletroencefalográfica entre os laudos dos examinadores.

Tabela 4 - Análise de concordância interexaminador 18 


\section{Lista de Figuras}

Figura 1 - Dinâmica da coleta de dados .......................................................... 20

Figura 2 - Fluxograma de seleção dos pacientes ................................................ 22 
1.1 Epidemiologia das Doenças Cerebrovasculares e de Eventos epilépticos elacionados.

1.2 O Papel do eletroencefalograma nas doenças cerebrovasculares.

1.3 Achados eletroencefalográficos gerais nas doenças cerebrovasculares..13

1.4 EEG em Sono em Pacientes Pós-AVC............................................ 15

1.5 Achados no Ataque Isquêmico Transitório...................................... 15

1.6 Achados no Acidente Vascular Cerebral Isquêmico............................. 15

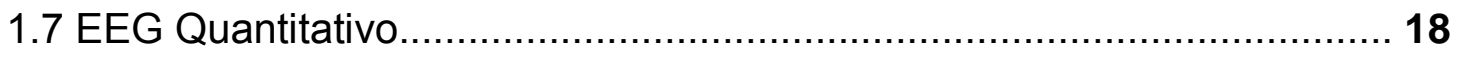

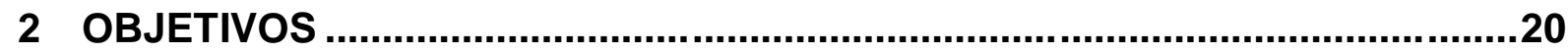

3 PACIENTES E MÉTODOS.......................................................................... 21

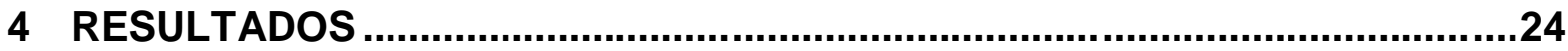

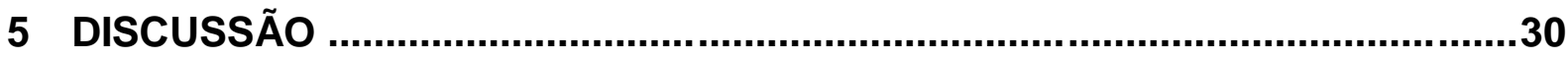

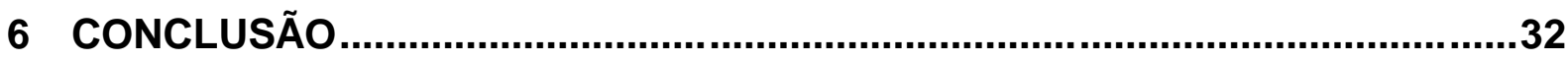

REFERÊNCIAS BIBLIOGRÁFICAS ............................................................... 33

APÊNDICE A ñ TERMO DE CONSENTIMENTO .................................................38

APÊNDICE B ñ FICHA DE COLETA DE DADOS CLÍNICOS ...............................40

APÊNDICE C ñ FICHA DE COLETA DE DADOS DO EEG ................................41

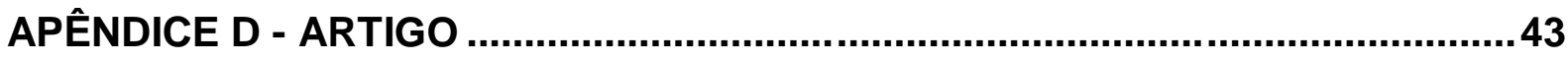

ANEXO A ñ TERMO DE CONCORDÂNCIA DE PESQUISA ..............................62

ANEXO B ñ DOCUMENTAÇÃO FEPECS/SES/DF .......................................64 


\section{INTRODUÇÃo}

\subsection{Epidemiologia das Doenças Cerebrovasculares e de Eventos epilépticos relacionados.}

As doenças cerebrovasculares constituem a terceira maior causa de mortalidade e a principal causa de incapacidade no mundo ${ }^{1}$. Apesar da tendência de queda na mortalidade nos últimos anos, o Brasil, entre todos os países da América Latina, é o que ainda apresenta as maiores taxas de mortalidade por acidente vascular cerebral $(\mathrm{AVC})^{2}$.

Em 2005 o acidente vascular cerebral isquêmico (AVCi) isoladamente foi responsável por $10 \%$ de todas as mortes (90006 mortes) e $10 \%$ de todas as admissões em hospitais públicos, sendo a principal causa de morte e incapacidade no Brasil ${ }^{3}$. A ocorrência de crises epilépticas e epilepsia sintomática secundária às doenças cerebrovasculares estão entre as possíveis sequelas levando a importante incapacidade para o trabalho e redução da qualidade de vida.

Em trabalhos prévios foram encontradas taxas de $4-10 \%$ de crises epilépticas secundárias a causas vasculares ${ }^{4}$ e de 2,6-30\% para epilepsia sintomática como sequela do evento índice ${ }^{5-7}$. Além disso, as doenças cerebrovasculares foram identificadas como principal causa de novas crises epilépticas em idosos ${ }^{8-10}$.

Importantes estudos também identificaram que no acidente vascular cerebral isquêmico os principais fatores de risco para crises epilépticas foram: localização cortical, lesões extensas ou múltiplas e incapacidade pós ictus, sendo o início tardio de crises (>2 semanas após o ictus) o principal fator de risco para epilepsia. Não foi encontrada correlação consistente entre a etiologia do $\mathrm{AVCi}$ e o início de crises epilépticas ${ }^{5,7}$.

\subsection{O Papel do eletroencefalograma nas doenças cerebrovasculares.}

Segundo dados da literatura o eletroencefalograma tem uma maior aplicação na avaliação de distúrbios funcionais provocados por doença cerebrovascular, sobretudo na deteç̧ão de focos epileptogênicos ${ }^{11}$. Além disso é útil para avaliação 
do prognóstico e manejo do HSAe no contexto de monitorização contínua com análise quantitativa em ambiente de unidade de terapia intensiva ${ }^{12}$.

O EEG é capaz de refletir mudanças dinâmicas no fluxo sanguíneo e metabolismo dentro de segundos e estimar a viabilidade tecidual de maneira tão sensível quanto as obtidas pelas imagens ponderadas por perfusão na imagem por ressonância magnética (IRM). Evidências em estudos com animais e humanos indicam que a cascata isquêmica é rápida, com alterações marcadas ocorrendo de segundos a minutos e alterações irreversíveis ocorrendo dentro de poucas horas ${ }^{11}$. A sequência DWI (diffusion weighted) da IRM e o EEG detectam respostas fisiopatológicas dentro da faixa entre $30-40 \mathrm{ml} / 100 \mathrm{~g} /$ minuto, sugerindo que os estágios de gradientes isquêmicos são comparavéis em suas sensibilidades ${ }^{13}$.

A acurácia do EEG de escalpo pode ser prejudicada pela amostragem do campo elétrico. Simulações biofísicas tem mostrado que a frequência espacial do EEG de escalpo requer densidades de amostragem mais altas para evitar deformações artificiais dos registros causadas por subamostragem ${ }^{14,15}$. Um estudo com pacientes com AVC isquêmico agudo, entre 8-36 horas do início dos sintomas, usando uma montagem com 128 canais, mostrou que uma acurada descrição topografica das alterações relacionadas ao AVC depende de uma adequada amostragem espacial do escalpo, sendo atingidas somente em registros com amostragem de 64 ou 128 canais $^{16}$. Quando a densidade cai a 32 canais, há distorção do espectro do EEG aumentando o erro de localização da região afetada.

Os resultados também mostraram que o EEG adquirido por 19 canais é clinicamente útil se os registros forem revisados por um especialista, mas as informações de topografia seriam limitadas. Como na prática diária seria inviável realizar aquisições de rotina em 128 canais, novos estudos com EEG convencional de 19 canais estão sendo feitos e são necessários para novas evidências que suportem o uso do EEG em pacientes com AVC.

Atualmente o EEG tem um papel limitado no manejo dos pacientes com AVC. Nas primeiras 48-72 horas, o grau de alentecimento focal geralmente se correlaciona com o déficit clínico ${ }^{17,18}$. Piora clínica como um resultado da progressão do AVC ou o desenvolvimento de edema e efeito de massa é acompanhado e às 
vezes precedido, por deterioração na atividade EEG. Marcada lentificação hemisférica após AIT, sobretudo se TCC e IRM normais, significam fluxo sanguíneo residual ou isquemia crônica adicionando urgência a avaliação e a consideração de intervenção cirúrgica ou endovascular ${ }^{17}$. Da mesma forma, a presença de ritmos lentos na faixa delta e ausência de ritmos fisiológicos ou rápidos em pacientes após grande AVC de território da artéria cerebral média (ACM) poderiam sugerir a evolução para infarto maligno e selecionar os pacientes que necessitariam de abordagem cirúrgica ${ }^{19}$.

Monitorização eletroencefalográfica durante a endarterectomia também pode ser útil pelo fato das alterações eletroencefalográficas aparecerem antes que a lesão isquêmica irreversível ocorra. Se prolongado ou severo, tais alterações são fortemente associadas a déficits clínicos pós-operatórios ${ }^{20}$. Monitorização eletroencefalográfica também pode ajudar a distinguir entre causas isquêmicas de causas epilépticas em disfunções neurológicas episódicas. Descargas epileptiformes, incluindo PLEDs são altamente correlacionados com crises epilépticas durante as primeiras semanas pós AVC e poderiam também acrescentar informações para predizer quais pacientes estariam sob maior risco de desenvolver epilepsia sintomática secundária à doença cerebrovascular ${ }^{21}$. Finalmente, o EEG é essencial para o diagnóstico diferencial de quadros sugestivos da Síndrome de Locked-In ${ }^{17}$.

\subsection{Achados Eletroencefalográficos gerais nas doenças cerebrovasculares.}

Vários estudos descreveram as alterações encontradas em pacientes portadores de doenças cerebrovasculares. Podem ser divididas em alterações do ritmo de base e alteracões focais ${ }^{22,23}$.

\subsubsection{Alterações no ritmo de base.}

Entre os padrões detectados notou-se que a preservação do ritmo cerebral de fundo rápido, a ausência de atividade delta e a presença de atividade teta ou mais rápida no foco isquêmico são preditores de um curso mais benigno ${ }^{24}$. Em contraposição, a lentificação difusa ou focal na faixa delta e a deterioração dos 
ritmos rápidos da atividade elétrica cerebral de base poderiam indicar um pior prognóstico com curso mais maligno ${ }^{19,25}$. Finalmente, foi encontrada relação entre lentificação da atividade de base e nível de consciência e prognóstico em 21 $\operatorname{dias}^{22,26}$.

\subsubsection{Alterações focais.}

Outros achados descritos são as atividades periódicas, atividade delta polimórfica (PDA), atividades rítmicas e atividade epileptiforme. Por causa da incidência e prevalência elevadas, o acidente vascular isquêmico é considerado a causa mais comum de atividade delta polimórfica focal contínua (PDA) ${ }^{27}$.

Em relação às atividades rítmicas, o FIRDA (Frontal Intermitent Rithymic Delta Activity) é comum e pode paradoxalmente ser de maior voltagem contralateralmente devido a atenuação no hemisfério cerebral isquêmico ${ }^{23}$.

Outra alteração relacionada com os acidentes vasculares cerebrais são as atividades periódicas. Anteriormente descritas como PLEDs (Periodic Lateralized Epileptiform Discharges), GPEDs (Generalized Periodic Epileptiform Discharges) sofreram uma modificação em sua terminologia em 2005, sendo revisadas em $2012^{28}$. Atualmente denominadas LPDs (Lateralized Periodic Discharges) e GPDs (Generalized Periodic Discharges), consistem em grafoelementos com morfologia bi ou trifásicas que ocorrem em intervalos regulares, periódicos ou quase periódicos. Em geral não ocorrem em pacientes com epilepsia crônica, mas podem ocorrer em desordens inflamatórias, infecciosas, tóxico-metabólicas, neurovasculares e no contexto de outras agressões agudas ao $\mathrm{SNC}^{29}$. Quando presentes, foram associados a crises epilépticas em até $90 \%$ dos pacientes ${ }^{11}$. Apesar disso, ainda há controvérsias sobre a correlação deste achado com a ocorrência de epilepsia ${ }^{25,30}$.

Atividade epileptiforme com morfologia de ondas agudas, pontas, polipontas não são usualmente encontradas em pacientes com AVC em fase aguda, porém podem evoluir com o tempo para epilepsia sintomática secundária a doença cerebrovascular ${ }^{11}$. 


\subsection{EEG em Sono em Pacientes Pós-AVC.}

Após infartos hemisféricos, foi descrita uma menor eficiência do sono, bem como menores quantidades de sono de ondas lentas e REM (rapid eyes moviment $)^{31,32}$. Lesões na porção ventrobasal do tálamo podem atenuar e alterar a frequência de fusos do sono ${ }^{33}$, embora lesões extra-talâmicas também possam alterar a atividade dos fusos do sono ${ }^{32}$. Em alguns trabalhos, a preservação da eficiência do sono, dos fusos e da quantidade de sono REM tem se correlacionado com bom prognóstico em pacientes com avc na fase aguda ${ }^{34,35}$.

\subsection{Achados no Ataque Isquêmico Transitório.}

O EEG em geral é normal nos pacientes com Ataques Isquêmicos Transitórios (AIT $)^{36}$. Entretanto se o exame for realizado durante ou em proximidade ao período sintomático, frequentemente observar-se-á lentificação focal no território

vascular $^{37,38}$. Isquemia crônica no território da artéria cerebral media está associado com atividade teta ou delta intermitente de baixa voltagem, principalmente sobre as regiões temporais ${ }^{39,40}$. Quando a atividade de ondas lentas é pronunciadamente lateralizada e de início recente, mas o paciente não apresenta qualquer sinal ou sintoma, existe o risco de oclusão vascular e subsequente infarto ${ }^{17}$.

\subsection{Achados no Acidente Vascular Cerebral Isquêmico.}

Em pacientes com oclusão aguda da artéria carótida interna $(\mathrm{ACl})$ ou da $\mathrm{ACM}$ ocorre uma atenuação difusa da atividade elétrica cerebral durante as primeiras horas $^{23}$. Atividade delta polimórfica aparece com máxima projeção sobre as regiões temporais anterior e media e em menor extensão sobre a região frontal. O ritmo alfa pode estar atenuado ou mostrar redução da reatividade ipsilateral ao $\mathrm{AVC}^{17}$.

Os preditores eletroencefalográficos de bom prognóstico em infartos de ACM 
são: preservação de ritmos de base fisiológicos como alfa e mu, além de ritmos rápidos ipsilaterais. Os de mau prognóstico são: perda de atividade rápida de fundo (alfa e beta) e grafoelementos fisiológicos e presença de atividade delta polimórfica persistente ipsilateral à lesão. Ritmo de fundo levemente normal ou levemente alterado e presença de ritmos lentos na faixa delta e teta não persistentes não mostraram correlação consistente com pior prognóstico clínico medido pelas escalas funcionais $^{18}$.

FIRDA (Frontal Intermitent Rithymic Delta Activity) é comum e pode paradoxalmente ser de maior voltagem contralateralmente devido a atenuação no hemisfério cerebral isquêmico. Grandes infartos são acompanhados de edema cerebral e efeito de massa que pode aumentar nas primeiras 72 horas levando a alentecimento dos ritmos cerebrais difusamente, com predomínio no hemisfério cerebral ipsilateral a lesão. LPDs e outras descargas epileptiformes ocorrem na minoria dos pacientes. Estas alterações agudas tendem a diminuir e desaparecer ao longo de poucas semanas ${ }^{23}$.

Durante a evolução, a atividade lenta polimórfica torna-se intermitente e mais frequências teta aparecem enquanto a atividade delta diminui. Frequências mais rápidas e toda a complexa atividade de fundo reaparece na extensão do córtex viável.

AVCs envolvendo as artérias cerebrais anteriores são menos comuns que aqueles envolvendo a ACM. O EEG nestes casos demonstra atividade delta sobre a area frontal ipsilateral, normalmente com atenuação de frequências mais rápidas da atividade de fundo. Infartos em território da artéria cerebral posterior produzem alentecimento focal que é máximo sobre as regiões temporal, parietal e occipital ipsilaterais. Há normalmente uma perda completa ou marcada perturbação do ritmo alfa do mesmo lado.

AVCs não complicados ou outras lesões da ponte inferior e medula, não são acompanhadas por anormalidades no $E_{E G}{ }^{17}$. Lesões da ponte rostral e mesencéfalo tem efeitos variáveis no EEG, largamente dependentes da extensão do dano ao sistema ativador reticular ascendente (SARA). Lesões pontinas anteriores poupando o tegmento pontino produzem a Síndrome Locked-in, na qual o paciente encontra-se 
mudo e tetraplégico, porem com consciência preservada e EEG normal devido o SARA, presente no tegmento, ter sido poupado ${ }^{41}$.

Quando há envolvimento do SARA na ponte rostral, mesencéfalo ou tálamo, os pacientes são comatosos e seus EEGs mostram vários tipos de anormalidades difusas da atividade de base, incluindo frequências rítmicas e arrítmicas, ondas teta e delta paroxísticas difusas e reatividade anormal ou ausente a vários estímulos ${ }^{42}$. Raramente, lesões do tronco rostral mostram atividade monorrítmica na frequência alfa difusamente que é uma variação do padrão coma alfa ${ }^{43}$.

Infartos lacunares únicos ou outras pequenas lesões subcorticais normalmente produzem poucas ou nenhuma alteracão no EEG agudamente. Somente $9 \%{ }^{44}$ a $13 \%{ }^{45}$ dos infartos lacunares são acompanhados por atividade lenta ipsilateral. Embora $53 \%$ dos pacientes com infartos lacunares tenham leves anormalidades, a maioria destes reflete AVCs prévios ou são inexplicados ${ }^{45}$.

Infartos em zonas fronteiriças (Watershed Ischemia ou Borderzone Strokes), decorrentes de hipoperfusão sistêmica, ocorrem mais comumente em pacientes idosos com história de alterações cardiovasculares crônicas e arteriosclerose cerebral com perda da auto-regulação do fluxo sanguíneo. Apresentam lesões no limite entre as áreas perfundidas pela $A C M$ e artéria cerebral anterior (ACA) ou entre aquelas perfundidas pela $\mathrm{ACM}$ e a artéria cerebral posterior (ACP). Os fatores predisponentes mais comuns são infecções, desidratação, diabetes mellitus, descompensação cardiovascular, anemia severa, insuficiência hepática com encefalopatia portocava e hipertensão severa. Os achados EEGs mais comuns são um alentecimento com um misto de frequências na faixa delta-teta, severa desorganização da atividade de base difusamente, com predomínio ipsilateral à lesão. Pode ocorrer atividade periódica lateralizada (LPDs) sobre as regiões posterotemporo-occipito-parietal ${ }^{11}$. Alguns autores sugerem que a presença de atividade periódica pode decorrer da hiperexcitabilidade nas zonas de penumbra de um AVC isquêmico ${ }^{25,46}$. 


\subsection{EEG Quantitativo.}

Análise de frequência e EEG por mapeamento topográfico pode ser superior ao EEG de rotina em detectar e localizar anormalidades subsequentes ao AVC. Esses métodos também parecem se correlacionar de maneira mais próxima ao fluxo sanguíneo cerebral regional ${ }^{47-49}$. Diversos estudos também tem mostrado boa correlação com o prognóstico clínico pós-AVC isquêmico, sobretudo em pacientes com maior grau de incapacidade (Rankin Scale grau 4 ou 5$)^{18}$.

Um estudo com 110 pacientes com AVC isquêmico na fase subaguda, avaliou os dados do EEG quantitativo pesquisando os desfechos de incapacidade medida pela escala de Rankin modificada ( $\mathrm{mRS}$ ), dependência e morte em 6 meses. Os resultados apontaramm que os dados do EEG quantitativo, tais como o Brain Symmetry Index (pdBSI) e o (delta + theta)/(alpha + beta) ratio (DTABR), foram significativamente correlacionados com o escore na mRS em 6 meses. A escala National Institute of Health Stroke Scale (NIHSS) e o pdBSI correlacionaram-se independententemente com a incapacidade em 6 meses. A dependência foi indicada independentemente pelas escalas NIHSS e pela DTABR enquanto a mortalidade foi indicada independentemente pelas variáveis idade no início do AVC, NIHSS e DTABR. Os achados indicam que o EEG na fase subaguda pode ser de valor prognóstico para avaliação de incapacidade, dependência e morte após 6 meses, sendo relevante para o planejamento da reabilitação, visando a diminuição de sequelas em longo prazo $^{50}$.

Em outro estudo foram avaliados 34 pacientes vítimas de hemorragia subaracnóidea, sob risco de isquemia tardia relacionada ao vasoespasmo cerebral, sendo avaliadas várias medidas fornecidas pelo EEG contínuo com análise quantitativa. Os resultados mostraram que a taxa alfa/delta (alfa power/delta power; ADR) demonstrou-se útil na detecção de isquemia secundária ao vasoespasmo definindo valores de corte para a redução da ADR a partir das quais havia um aumento do risco de desenvolvimento de isquemia secundária ao vasoespasmo. Amensuração da queda da ADR permitiu avaliar este risco com sensibilidade de $100 \%$ e especificidade de $76 \%$, apontando tratar-se de uma ferramenta que propicia 
detecção e possibilidade de intervenção precoce, permitindo a prevenção de infartos e redução da morbidade neurológica na hemorragia subaracnóidea ${ }^{12}$.

Apesar da introdução de padronizações da técnica de aquisição, leitura e nomenclatura, a interpretação do EEG permanece sendo subjetiva, variando de acordo com as características individuais dos eletroencefalografistas o que pode afetar as taxas de concordância interobservador ${ }^{51}$.

O presente estudo visa avaliar o perfil eletroencefalográfico de pacientes vítimas de acidente vascular cerebral isquêmico durante a fase aguda atendidos em um hospital terciário do Distrito Federal bem como avaliar o grau de concordância interobservador dos registros realizados. 


\section{OBJETIVOS}

1) Identificar os achados eletroencefalográfico de um grupo de pacientes com AVC isquêmico nas fases aguda a subaguda atendidos em um hospital terciário da rede de saúde pública do Distrito Federal;

2) Avaliar a taxa de concordância interobservador da análise visual dos eletroencefalogramas de pacientes com AVC isquêmico na fase aguda a subaguda. 


\section{PACIENTES E MÉTODOS}

Trata-se de um estudo observacional, analítico, transversal, destinado a avaliação do grau de concordância interobservador.

Foram admitidos no estudo os pacientes maiores de 18 anos, com quadro inédito de Acidente Vascular Cerebral Isquêmico em fase aguda em um hospital público terciário da cidade de Brasília-DF, Brasil, no período de outubro de 2014 a julho de 2015, submetidos a avaliação pela equipe de neurologistas daquele hospital e que tivessem realizado ao menos um exame de neuroimagem (tomografia de crânio não contrastada de 32 canais ou ressonância magnética de crânio 1,5 Teslas), submetidos ou não ao procedimento de trombólise endovenosa com alteplase (r-TPA) e que tiveram ao menos um registro eletroencefalográfico considerado tecnicamente satisfatório realizado até sete dias após o início do quadro clínico do AVC isquêmico. As informações sobre os dados clínicos e de neuroimagem dos pacientes foram obtidos do prontuário eletrônico em revisão.

Foram excluídos os pacientes com histórico de abordagem neurocirúrgica, epilepsia pré-existente, diagnóstico clínico, laboratorial e ou radiológico de doenças que poderiam mimetizar um quadro de acidente cerebrovascular isquêmico ou alterar a acurácia das medidas realizadas, tais como: traumatismo cranioencefálico, hemorragia subaracnóidea espontânea, trombose venosa cerebral, distúrbios hidroeletrolíticos ou metabólicos graves, doenças neurodegenerativas, história de acidentes vasculares cerebrais prévios ou de outras comorbidades que pudessem alterar a acurácia das medidas realizadas.

A amostragem foi realizada por conveniência. Foram selecionados todos os pacientes que preenchiam os critérios de inclusão da pesquisa e que puderam ser avaliados clinicamente e pelos exames complementares eletroencefalograma e avaliação por neuroimagem (tomografia de crânio e ou Ressonância Magnética de Crânio).

O eletroencefalograma foi realizado com aparelho de 21 canais, com eletrodos de superfície (escalpo) colocados de acordo com o sistema internacional 10-20, analisado pelas montagens bipolares e referenciais comuns, sob banda de filtro de 1,0 a $70,0 \mathrm{~Hz}$ e com sensibilidade de 7 microvolts por milímetro, conforme 
recomendações da Academia Americana de Neurofisiologia Clínica/Sociedade Brasileira de Neurofisiologia Clínica $(\mathrm{SBNC})^{52}$.

Os registros foram interpretados por meio de análise visual e independente. Os casos foram distribuídos de forma aleatória entre dois ou três eletroencefalografistas experientes com titulação em neurofisiologia clínica. Os avaliadores eram informados sobre o diagnóstico de AVC isquêmico dos pacientes, porém não possuíam conhecimento sobre exame clínico, gravidade do quadro ou se os pacientes haviam sido submetidos ou não à trombólise endovenosa. A Figura 1 resume as etapas da coleta de dados.

\section{Figura 1. Dinâmica da coleta de dados.}

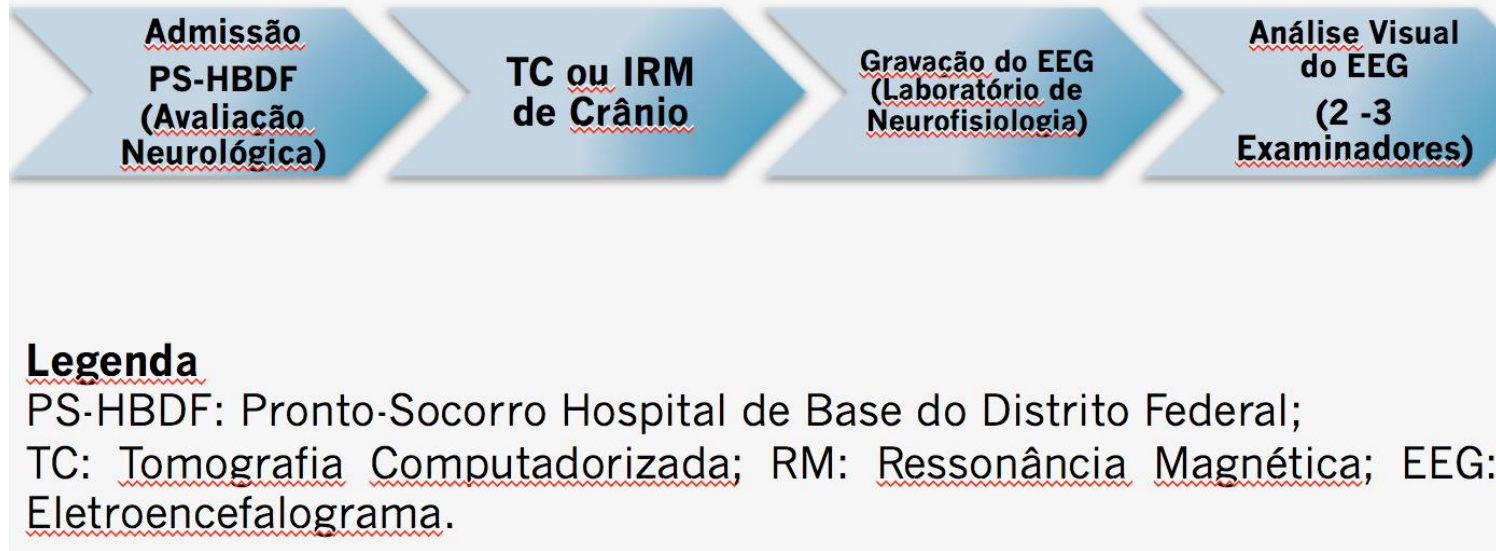

A análise dos traçados foi orientada por um formulário padronizado contendo as seguintes questões: qualidade técnica de gravação, organização e simetria da atividade elétrica cerebral de base, presença e tipo de atividade lenta (contínua versus intermitente), presença de atividade epileptiforme, lateralização dos achados anormais e conclusão do exame (normal versus anormal).

A análise estatística descritiva e a análise de concordância pelo teste kappa foram realizadas com o auxílio do software IBM SPSS Statistics ${ }^{\circledR}$ versão 22 . Foram consideradas as seguintes faixas de valores para classificação do grau de concordância pelo coeficiente de kappa: valores $\leq 0$ indicando ausência de concordância, 0.01-0.20 como nenhuma a leve concordância, $0.21-0.40$ como pobre, 0.41 - 0.60 como moderada, 0.61 - 0.80 como substancial e $0.81-1.00$ como quase perfeita ${ }^{53}$. 
As variáveis da pesquisa utilizadas para análise de concordância foram: assimetria da atividade de base, organização da atividade de base, presença de atividade lenta, presença de atividade epileptiforme, achados anormais lateralizatórios e conclusão do exame.

A presente pesquisa foi aprovada pelo parecer com número CAAE FEPECS 33843314.2.0000.5553 e do comitê de ética local do Hospital de Base do Distrito Federal conforme Anexos A e B. Todos os pacientes preencheram o termo de consentimento livre e esclarecido. 


\section{RESULTADOS}

Foram avaliados 44 pacientes dos quais 5 foram excluídos por terem histórico de $A V C$ isquêmico prévio ou diagnóstico de $A V C$ hemorrágico à admissão. Sete pacientes foram excluídos por terem a qualidade de seus registros eletroencefalográficos considerada insatisfatória por qualquer um dos três eletroencefalografistas e 14 por terem os seus registros eletroencefalográficos sido avaliados apenas por um dos dois examinadores cujos dados foram utilizados nas análises estatísticas. A amostra final foi composta por 18 pacientes. A Figura 2 demonstra o fluxograma para seleção dos pacientes do estudo.

\section{Figura 2. Fluxograma de selecão dos pacientes.}

\section{Total \\ (44)}

AVC prévio ou

AVCH primário

(5)

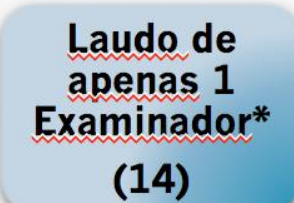

Amostra

Final

(18)

Para análise descritiva da amostra foram utilizados os laudos dos pacientes realizados pelo Examinador 1, o qual foi arbitrariamente escolhido pelo fato de ter realizado o maior número de avaliações dentre os 3 examinadores. Para a análise 
comparativa entre observadores foram utilizados os dados dos pacientes avaliados pelos Examinadores 1 e 2 . A escolha foi também realizada de forma arbitrária e baseou-se na presença do maior número de pacientes avaliados concomitantemente por dois examinadores.

$\mathrm{Na}$ Tabela 1 estão descritas as características demográficas, clínicas e os achados de neuroimagem da amostra. Os pacientes apresentaram média de idade de 50 anos, sendo que a maioria não foi submetida à trombólise endovenosa. Nenhum paciente teve crises epilépticas clinicamente detectadas até o momento da realização do eletroencefalograma.

Todos os pacientes apresentaram lesões detectáveis à tomografia computadorizada de crânio (TCC) ou à Ressonância Magnética de Crânio. Três pacientes apresentaram TCC normais na admissão sendo submetidos a um novo exame de neuroimagem confirmatório durante a internação. As lesões identificadas foram classificadas quanto à sua lateralização, não sendo, entretanto, classificadas quanto à topografia ou profundidade. 
Tabela 1. Características clínicas e demográficas da amostra.

\begin{tabular}{llll}
\hline Característica & Categoria & $\mathrm{n}$ & $\%$ \\
\hline Idade & Mínima & 21 & \\
& Máxima & 77 & \\
& Média & 50 & \\
Sexo & Feminino & 9 & $50 \%$ \\
& Masculino & 9 & $50 \%$ \\
Trombólise Endovenosa & Sim & 5 & $28 \%$ \\
& Não & 13 & $72 \%$ \\
Craniectomia & Sim & 1 & $6 \%$ \\
\multirow{4}{*}{ Crise Epiléptica } & Não & 17 & $94 \%$ \\
& Sim & 0 & $0 \%$ \\
Lesão à Neuroimagem+ & Não & 18 & $100 \%$ \\
& Sim & 18 & $100 \%$ \\
Lateralização à neuroimagem & Não & 0 & $0 \%$ \\
& Esquerda & 11 & $61 \%$ \\
& Direita & 6 & $33 \%$ \\
& Bilateral & 1 & $6 \%$ \\
\hline
\end{tabular}

Legenda: $\mathrm{EEG}=$ eletroencefalograma; Delta Ictus-EEG= intervalo de tempo entre início do quadro isquêmico até a realização do EEG; += Tomografia computadorizada de crânio e ou Ressonância Magnética de Crânio.

Fonte: Dados da pesquisa 2015.

A análise dos dados do EEG revelou que o intervalo de tempo entre o ictus vascular e a realização do eletroencefalograma variou de 0 a 7 dias com média de 3,9 dias. A duração da gravação dos registros variou de 21 a 41 minutos com média de 33,8 minutos. À partir da análise dos laudos realizados pelo Examinador 1, obtivemos os resultados descritos a seguir

Cerca de $28 \%$ dos pacientes apresentaram uma atividade elétrica cerebral assimétrica, ou seja, traçados que apresentaram uma diferença significativa de amplitude ou de frequência dos ritmos da atividade elétrica cerebral. Metade dos pacientes apresentou critérios para a desorganização da atividade elétrica cerebral de base. Foram classificados como tendo atividade elétrica cerebral de base desorganizada os exames que preencheram ao menos um dos seguintes critérios: lentificação do ritmo posterior (menor ou igual a $8,0 \mathrm{~Hz}$ ), ausência ou redução da 
presença dos ritmos rápidos em regiões anteriores ou redução da amplitude dos ritmos elétricos cerebrais (menor que $10 \mathrm{mcV} / \mathrm{mm}$ ).

Foi observada a presença de atividade lenta anormal na faixa delta e ou teta em cerca de $61 \%$ dos pacientes. Destes, pouco mais da metade (54\%) apresentaram atividade lenta focal intermitente e os demais apresentaram atividade lenta anormal contínua. Apenas 39\% dos pacientes não apresentaram regiões de lentificação focal em seus traçados.

Dos 18 pacientes analisados, apenas dois apresentaram grafoelementos epileptiformes (ondas agudas e espículas) em seus traçados. Entretanto, não foi relatada atividade epiléptica clínica pelos médicos assistentes em nenhum dos pacientes. Anormalidades unilaterais, ou seja, achados lateralizatórios, foram percebidos em $61 \%$ dos pacientes, enquanto o restante apresentou achados bilaterais ou exames normais.

O eletroencefalograma foi laudado como anormal em cerca de $78 \%$ dos pacientes estudados. Nestes, houve a presença de ao menos um dos seguintes critérios: presença de desorganização ou assimetria da atividade elétrica cerebral de base, presença de atividade lenta focal ou generalizada, presença de grafoelementos epileptiformes e a presença de outros grafoelementos anormais. A caracterização dos achados eletroencefalográficos realizada pelo examinador 1 encontra-se resumida na Tabela 2. 


\begin{tabular}{llll}
\hline Tabela 2. Características Eletroencefalográficas da amostra*. & & \\
\hline & & \multicolumn{2}{l}{ Examinador 1} \\
Característica & Categoria & $\mathrm{n}$ & $\%$ \\
\hline Delta Ictus-EEG (em dias) & Mínimo & 0 & \\
& Máximo & 7 & \\
Duração do EEG (em minutos) & Média & 3,9 & \\
& Mínima & 21 & \\
Simetria da atividade de base & Máxima & 41 & \\
& Média & 33,8 & \\
Organização da atividade de base & Simétrica & 13 & $72 \%$ \\
Presença de atividade lenta anormal & Assimétrica & 5 & $28 \%$ \\
& Organizada & 9 & $50 \%$ \\
Atividade lenta anormal (tipo) & Desorganizada & 9 & $50 \%$ \\
& Sim & 7 & $39 \%$ \\
& Não & 11 & $61 \%$ \\
Atividade epileptiforme & Ausente & 7 & $39 \%$ \\
Lateralização & Intermitente & 6 & $33 \%$ \\
& Contínua & 5 & $28 \%$ \\
Conclusão do exame & Sim & 2 & $11 \%$ \\
& Não & 16 & $89 \%$ \\
& Direita & 3 & $17 \%$ \\
& Esquerda & 8 & $44 \%$ \\
& Não lateralizada & 7 & $39 \%$ \\
& Normal & 4 & $22 \%$ \\
& Anormal & 14 & $78 \%$ \\
\hline
\end{tabular}

Legenda:*Dados da análise do Examinador 1; EEG=eletroencefalograma; Delta Ictus-EEG= intervalo de tempo entre início do quadro isquêmico até a realização do EEG;

Fonte: Dados da pesquisa 2015.

À partir dos dados obtidos da avaliação dos traçados pelos examinadores $1 \mathrm{e}$ 2 foi realizada a avaliação do nível de concordância interexaminadores pelo teste kappa. Foram obtidos os seguintes resultados: a simetria da atividade elétrica cerebral de base, a presença de atividade lenta anormal (não especificada como contínua ou intermitente), a presença de atividade epileptiforme e a conclusão sobre a presença de qualquer anormalidade apresentaram concordância substancial entre os examinadores 1 e 2 . A avaliação da organização da atividade de base e da lateralização dos achados anormais pelo EEG apresentaram concordância apenas moderada. A Tabela 3 mostra os resultados das análises dos observadores 1 e 2 e a 
Tabela 4 resume os achados da análise da concordância interexaminador nesta amostra.

\begin{tabular}{llccccc}
\hline Tabela 3. Comparativo da atividade eletromiográfica entre os laudos do s Examinadores. \\
\hline \multirow{2}{*}{ Característica } & \multicolumn{2}{c}{ Examinador 1} & \multicolumn{3}{c}{ Examinador 2} \\
& Categoria & $\mathrm{n}$ & $\%$ & $\mathrm{n}$ & $\%$ \\
\hline Simétrica & Sim & 13 & $72 \%$ & 11 & $61 \%$ \\
Organizada & Nao & 5 & $28 \%$ & 7 & $39 \%$ \\
& Sim & 9 & $50 \%$ & 11 & $61 \%$ \\
Atividade lenta anormal & Não & 9 & $50 \%$ & 7 & $39 \%$ \\
& Sim & 11 & $61 \%$ & 6 & $33 \%$ \\
Atividade lenta anormal (tipo) & Não & 7 & $39 \%$ & 12 & $67 \%$ \\
& Ausente & 7 & $39 \%$ & 6 & $33 \%$ \\
& Intermitente & 6 & $33 \%$ & 5 & $28 \%$ \\
Atividade epileptiforme & Contínua & 5 & $28 \%$ & 7 & $39 \%$ \\
\multirow{4}{*}{ Lateralização } & Sim & 2 & $11 \%$ & 1 & $6 \%$ \\
& Não & 16 & $89 \%$ & 17 & $94 \%$ \\
& Direita & 3 & $17 \%$ & 3 & $17 \%$ \\
Conclusão do exame & Esquerda & 8 & $44 \%$ & 9 & $50 \%$ \\
& Não lateralizada* & 7 & $39 \%$ & 6 & $33 \%$ \\
& Normal & 4 & $22 \%$ & 4 & $22 \%$ \\
& Anormal & 14 & $78 \%$ & 14 & $78 \%$ \\
\hline
\end{tabular}

Legenda: EEG=eletroencefalograma, ${ }^{*}=E E G$ normal ou anormalidades bilaterais.

Fonte: Dados da pesquisa 2015.

\begin{tabular}{cccc}
\hline \multicolumn{4}{c}{ Tabela 4. Análise de concordância interexaminador. } \\
\hline Critério Analisado & Nível de Concordância E1 x E2 & Kappa & Significância \\
Assimetria da Atividade de Base & Substancial & 0,753 & $\mathrm{p}=0,001$. \\
Organização da Atividade de Base & Moderada & 0,556 & $\mathrm{p}=0,016$ \\
Presença de Atividade Lenta & Substancial & 0,668 & $\mathrm{p}=0,00$ \\
Presença de Atividade Epileptiforme & Substancial & 0,64 & $\mathrm{p}=0,004$ \\
Achados lateralizatórios & Moderada & 0,463 & $\mathrm{p}=0,008$ \\
Conclusão do Exame & substancial & 0,679 & $\mathrm{p}=0,004$ \\
\hline
\end{tabular}

Legenda: E1= Examinador 1; E2=Examinador; Kappa=Teste de Concordância de Kappa; $\mathrm{p}=\mathrm{p}$ valor. 


\section{DISCUSSÃO}

No presente trabalho foi avaliada a concordância interexaminador do EEG em pacientes portadores de doença cerebrovascular e foram descritos os achados eletroencefalográficos de um grupo de pacientes com doença cerebrovascular isquêmica atendidos na fase aguda a subaguda em um hospital terciário do Distrito Federal. Este foi o primeiro estudo a comparar a taxa de concordância interobservador da análise visual do EEG em pacientes portadores de AVC.

Os sinais de EEG são complexos e a inspeção visual dos traçados para extrair características relevantes é sujeita a variabilidade individual ${ }^{54}$. Estudos prévios observaram uma taxa de concordância interexaminador variando amplamente de $19 \%{ }^{55}$ a $95 \%{ }^{56}$.

Embora não exista uma padronização internacional quanto aos critérios e classificação dos achados eletroencefalográficos, foi observada no presente estudo uma substancial concordância interexaminador para a maior parte dos critérios analisados. Isso pode ter ocorrido devido ao uso de categorias mais amplas de classificação, o que segundo estudos prévios $^{57}$ pode aumentar o grau de concordância quando comparada a categorias mais específicas como a presença de atividade epileptiforme.

Variações nas características relatadas dos testes podem ser relacionadas a diferenças nas características dos pacientes ou aos limiares dos examinadores ao interpretar os testes como positivos, sendo no último aspecto relacionado a variação de até $33 \%$ da acurácia do exame ${ }^{58}$.

É importante notar que apesar de ter havido uma concordância substancial entre os examinadores na maior parte dos quesitos analisados, nenhuma das características apresentou concordância quase perfeita. Além disso, é preciso destacar a limitação dos dados do presente estudo que pode ter originado um viés devido à amostra reduzida em relação aos achados de menor incidência como a presença de atividade epileptiforme identificada em apenas 2 pacientes.

A concordância apenas moderada em relação a organização da atividade elétrica cerebral de base e a lateralização dos achados anormais pode ter decorrido de dificuldades na padronização dos critérios de classificação eletroencefalográficos, 
sobretudo em relação à organização da atividade elétrica cerebral, também do número reduzido da amostragem ou ainda de características individuais dos avaliadores.

Concordamos com as recomendações de Gerber que propõe medidas para o aumento da concordância interobservador na avaliação do EEG, tais como (1) manter a simplicidade da terminologia, (2) limitar o uso de descritores opcionais, (3) aumentar as definições publicadas com exemplos visuais que ilustram os limites dos achados eletroencefalográficos a um termo individual adicionalmente aos protótipos, (4) desenvolver um treinamento interativo incluindo o teste dos participantes que participarão da pesquisa.

Recomenda-se a padronização da terminologia para aumentar o nível de concordância interexaminador, a confiabilidade e para maximizar a comunicação entre os diferentes revisores de eletroencefalogramas de um mesmo paciente ${ }^{54}$.

Como citado anteriormente, o trabalho possui algumas limitações tais como: o tamanho da amostra, o uso de amostragem não probabilística, a ausência de avaliação eletroencefalográfica quantitativa e a ausência de localização acurada das lesões (distinção entre lesões corticais e subcorticais e sua topografia) à neuroimagem.

São necessários estudos adicionais com maior amostragem e métodos de seleção aleatorizados para melhor caracterização do perfil eletroencefalográfico dos pacientes atendidos com acidente cerebrovascular no Distrito Federal.

Além disso, um estudo longitudinal prospectivo com maior amostragem, associada à avaliação de parâmetros clínicos e de dados quantitativos do eletroencefalograma poderia trazer dados adicionais sobre as características dos grupos tratados ou não com a trombólise endovenosa, sobre a aplicabilidade do eletroencefalograma e assim gerar avanços nos cuidados aos pacientes com doença cerebrovascular. 


\section{CONCLUSÃO}

Os dados deste estudo sugerem que o eletroencefalograma possui uma substancial concordância interexaminador em relação a alguns itens da avaliação, tais como assimetria da atividade de base, presença de atividade lenta, presença de atividade epileptiforme e conclusão do exame, entretanto são necessários novos estudos com maior número de amostragem e com uso de terminologia e critérios de classificação padronizados.

Tais medidas são necessárias para aumentar a concordância interexaminador, sobretudo para critérios mais complexos e amplos, tais como a avaliação da organização da atividade elétrica cerebral de base, levando a uma maior aplicabilidade do método no manejo do paciente portador de doença cerebrovascular. 


\section{REFERÊNCIAS BIBLIOGRÁFICAS}

1. Marsh JD, Keyrouz SG. Stroke prevention and treatment. J Am Coll Cardiol. 2010;56(9):683-91.

2. $\quad$ Lotufo P a. Stroke: a neglected disease in Brazil. São Paulo Med J. 2005;123(1):3-4.

3. Martins SCO, Freitas GR De, Pontes-Neto OMOM, Pieri A, Moro CHC, Jesus PAP De, et al. Guidelines for acute ischemic stroke treatment: part I. Arq Neuropsiquiatr [Internet]. 2012;70(11):885-93. Available from: http://ovidsp.ovid.com/ovidweb.cgi?T=JS\&PAGE=reference\&D=psyc7\&NEWS $=\mathrm{N} \& A N=2012-23551$ -

012\%5Cnhttp://www.ncbi.nlm.nih.gov/pubmed/23175203\%5Cnhttp://www.ncbi. nlm.nih.gov/pubmed/22899035

4. Baldin CF, Alexandrov A V, Bellavance A, Bornstein N, Chambers B, Coté R, et al. Seizures after stroke. Arch neurol [Internet]. 2000;57:1617-22. Available from: http://www.ncbi.nlm.nih.gov/pubmed/11074794

5. Chen T-C, Chen Y-Y, Cheng P-Y, Lai C-H. The incidence rate of post-stroke epilepsy: a 5-year follow-up study in Taiwan. Epilepsy Res [Internet]. 2012;102(3):188-94. Available from:

http://www.ncbi.nlm.nih.gov/pubmed/22749919

6. Romaniak A B-GM. Post-Stroke Epilepsy. Neurol Neurochir Pol. 1998;May$\operatorname{Jun}(32(3)): 603-13$.

7. Burn J, Dennis M, Bamford J, Sandercock P, Wade D, Warlow C. Epileptic seizures after a first stroke: the Oxfordshire Community Stroke Project. BMJ [Internet]. 1997;315(7122):1582-7. Available from:

http://www.pubmedcentral.nih.gov/articlerender.fcgi?artid=2127973\&tool=pmce ntrez\&rendertype=abstract

8. Hauser WA. Seizure disorders. Epilepsia. 1992;33(suppl 4:S6-14.

9. Luhdorf K, Jensen LK PA. Etiology of seizures in the elderly. Epilepsia. 1986;27:458-63.

10. Olsen T. Post-stroke epilepsy. Curr Arthroscler Rep. 2001;3:340-4.

11. Niedermeyer, E., Silva F.L., Tettenborn B. Cerebrovascular Diseases and EEG. In: Electroencephalography, Basic Principles, Clinical Applications, and Related Fields Williams and Wilkins, Philadelphia,PA, Sixth Edition. 2011. p. 351-74.

12. Claassen J, Hirsch LJ, Kreiter KT, Du EY, Connolly ES, Emerson RG MS. Quantitative continuous EEG for detecting delayed cerebral ischemia in patients with poor-grade subarachnoid hemorrhage. Clin Neurophysiol. 2004; $\operatorname{Dec}(115(12)): 2699-710$. 
13. Hossmann K. Viability thresholds and the penumbra of focal ischemia. Ann Neurol. 1994;36:557-65.

14. Genvins A, Le J, Brickett $P$ et al. Seeing through the skull: advanced EEG use MRIs to accurately measure cortical activity from the scalp. Brain Topogr. 1991;4:125-31.

15. Jackel, RA, Harner R. Computed EEG topography in acute stroke. Neurophysiol Clin. 1989;19:185-97.

16. Luu P, Tucker DM, Englander $R$ et al. Localizing acute stroke-related EEG changes: assessing the effects of spatial undersampling. J Clin Neurophysiol. 2001;18:302-17.

17. Ebersole, J. S., Pedley TA. Current practice of clinical electroencephalography. Philadelphia: Lippincott Williams \& Wilkins, 3a Edição, 2003. Capítulo 11. 304$11 \mathrm{p}$.

18. Cillessen JP, van Huffelen a C, Kappelle LJ, Algra A, van Gijn J. Electroencephalography improves the prediction of functional outcome in the acute stage of cerebral ischemia. Stroke [Internet]. 1994 Oct;25(10):1968-72. Available from: http://www.ncbi.nlm.nih.gov/pubmed/8091439

19. Burghaus L, Hilker R, Dohmen C, Bosche B, Winhuisen L, Galldiks N, et al. Early electroencephalography in acute ischemic stroke: Prediction of a malignant course? Clin Neurol Neurosurg. 2007;109(1):45-9.

20. Collice M, Arena O, Fontana RA et al. Role of EEG monitoring and crossclamping duration in carotid endarterectomy. J Neurosurg. 1986;65:815-9.

21. Holmes $\mathrm{G}$. The electroencephalogram as a predictor of seizures following cerebral infarction. Clin Electroencephalogr. 1980;11:83-6.

22. Kayser-Gatchalian MC NB. The prognostic value of EEG in ischaemic cerebral insults. Electroencephalogr Clin Neurophysiol. 1980;49:608-17.

23. Van der Drift JHA MO. The EEG in cerebral ischemic lesions. Correlations with clinical and pathological findings. In: In: Gastaut H, Meyer JS, eds Cerebral Annoxia and the Electroencephalogram Springfield IL: Thomas; 1961. p. 18096.

24. Van der Drift J.H.A. KNKD. The EEG in cerebrovascular disorders in relations to pathology. In: In: A REmond, Ed-in-chief Handbook of Electroencephalography and Clinical Neurophysiology Vol 14A Amsterdan: Elsevier; 1972. p. 12-130, 47-64.

25. Pohlmann-Eden B, Hoch DB CJ. Periodic Lateralized epileptiform discharges: a critical rewiew. JClin Neurophysiol. 1996;13:519-30.

26. Sainio K, Stenberg D KI. Visual and spectral EEG analysis in the evaluation of the outcome in patients with ischemic brain infarction. Electroencephalogr Clin Neurophysiol. 1983;56:117-24.

27. Joynt, RJ; Cape, CA; Knott J. Significance of focal delta activity in adult 
electroencephalogram. Arch Neurol. 1965;12:631-638.

28. Hirsch LJ, LaRoche SM, Gaspard N, Gerard E, Svoronos A, Herman ST, et al. American Clinical Neurophysiology Society's Standardized Critical Care EEG Terminology: 2012 version. J Clin Neurophysiol. 2013;30(1):1-27.

29. Gaches J. Les activtiés périodiques en EEG. Rev EEG Neurophysiol. 1971;1:9-33.

30. Fitzpatrick, W, Lowry N. PLEDS: clinical correlates. Can J Neurol Sci. 2007;34:443-50.

31. Müller C, Ackermann P BM. Visual and spectral analysis of sleep EEG in acute hemispheric stroke. Eur Neurol. 2002;48(164-171).

32. Gottselig JM, Bassetti CL AP. Power and coherence of sleep spindle frequency activity following hemispheric stroke. Brain. 2002;125:373-83.

33. Jasper H, Van Buren J. Interrelationships between cortex and subcortical structures: clinical electroencephalographic studies. Electroencephalogr Clin Neurophysiol Suppl. 1953;4:168-88.

34. Giubilei $F$, Iannilli M, Vitale A. Sleep patterns in acute ischemic stroke. Acta Neurol Scand. 1992;86:567-71.

35. Vock J, Ackermann P, Bischof M. Evolution of sleep and sleep EEG after hemispheric stroke. Brain. 2002;125:373-83.

36. Engel $\mathrm{S}$, Lechner $\mathrm{H}$, Logar $\mathrm{C}$. Clinical value of EEG in transient ischemic attacks. In: In: Lechner $\mathrm{H}$, Aranibar A, eds EEG and clinical neurophysiology Amsterdam: Excerpta Medica. 1980. p. 173-80.

37. Madkour $\mathrm{O}$, Elwan $\mathrm{O}$, Hamdy $\mathrm{H}$. Transient ischemic attacks: electrophysiological (conventional and topographic EEG) and radiological (CCT) evaluation. J Neurol Sci. 1993;119:8-17.

38. Marshall D, Brey R, Morse M. Focal and/or lateralized polymorphic delta activity: association with either anormal or nonfocal computed tomographic scans. Arch Neurol. 1998;45:33-5.

39. Bruens J, Gastaut H, Gioive G. Electroencephalographic study of the signs of chronic vascular insufficiency of the Sylvian region in aged people. Electroencephalogr Clin Neurophysiol. 1960;12:283-95.

40. Gastaut H, Bruens J, Roger J. Etude electroencephalographique des signes d'insuffisiance circulatoire syvienne chronique. Rev Neurol. 1959;100:59-65.

41. Markand O. EEG in the locked-in syndrome. Electroencephalogr Clin Neurophysiol. 1976;40:529-34.

42. Schaul N, Gloor P, Gotman J. The EEG in deep midline lesions. Neurology. 1981;31:157-67.

43. Westmoreland B, Klass D, Sharbrough F. Alpha coma: 
electroencephalographic, clinical, pathological and etiological correlation. Arch Neurol. 1998;32:713-8.

44. Macdonell R, Donnan G, Bladin P. The electroencephalogram and acute ischemic stroke. Arch Neurol. 1988;45:520-524.

45. Petty G, Labar D, Fisch B. Electroencephalography in lacunar infarction. J Neurol Sci. 1995;134:47-50.

46. Pohlmann-Eden B, Hoch O, Cochius J. Stroke and epilepsy: critical review of the literature. Part I: Epidemiology and risk factors. Cerebrovasc Dis. 1996;6:332-8.

47. Nagata K, Mizukami M, Araki G. Topographic electroencephalographic study of cerebral infarction using computed mapping of the EEG. J Cereb Blood Flow Metab. 1982;2:79-88.

48. Nagata K, Yunoki K, Araki G. Topographic electroencephalographic study of transient ischemic attacks. Electroencephalogr Clin Neurophysiol. 1984;58:291-301.

49. Nuwer M, Jordan S, Ahn S. Evaluation of stroke using EEG frequency analysis and topographic mapping. Neurology. 1987;37:1153-9.

50. Sheorajpanday RVA, Nagels G, Weeren AJTM, De Deyn PP. Quantitative EEG in ischemic stroke: Correlation with infarct volume and functional status in posterior circulation and lacunar syndromes. Clin Neurophysiol [Internet]. 2011;122(5):884-90. Available from: http://dx.doi.org/10.1016/j.clinph.2010.08.020

51. Gerber PA, Chapman KE, Chung SS, Drees C, Maganti RK, Ng Y-T, et al. Interobserver agreement in the interpretation of EEG patterns in critically ill adults. J Clin Neurophysiol. 2008;25(5):241-9.

52. Nuwer MR, Comi C, Emerson R, Fuglsang-Frederiksen a., Gu'erit JM, Hinrichs $\mathrm{H}$, et al. IFCN standards for digitial recording of clinical EEG.

Electroencephalogr Clin Neurophysiol. 1998;106:259-61.

53. McHugh M. Interrater reliability: the kappa statistic. Biochem Medica [Internet]. 2012;22(3):276-82. Available from:

http://hrcak.srce.hr/index.php?show=clanak\&id_clanak_jezik=132393

54. Tatum WO, Olga S, Ochoa JG, Munger Clary H, Cheek J, Drislane F, et al. American Clinical Neurophysiology Society Guideline 7: Guidelines for EEG Reporting. J Clin Neurophysiol [Internet]. 2016;33(4):328-32. Available from: http://content.wkhealth.com/linkback/openurl?sid=WKPTLP:landingpage\&an=0 0004691-20160800000009\%5Cnhttp://www.ncbi.nlm.nih.gov/pubmed/27482790

55. Williams GW, Lüders HO, Brickner A, Goormastic M, Klass DW. Interobserver variability in EEG interpretation. Neurology [Internet]. 1985 Dec;35(12):1714-9. Available from: http://www.ncbi.nlm.nih.gov/pubmed/4069362 
56. Struve FA, Dorothy I, Green MA, Howard A. Reliability of Clinical Interpretation of the Electroencephalogram. 1975;6(2):54-60.

57. Gilbert DL, Sethuraman G, Kotagal U, Buncher CR. Meta-analysis of EEG test performance shows wide variation among studies. Neurology. 2002;60:564-70.

58. Deeks J. Systematic reviews of evaluations of diagnostic and screening tests. Bmj [Internet]. 2001;323(7305):157-62. Available from: http://www.bmj.com/content/323/7305/157.1.full

59. Jordan KG. Emergency EEG and continuous EEG monitoring in acute ischemic stroke. J Clin Neurophysiol [Internet]. 2004;21(5):341-52. Available from: http://www.ncbi.nlm.nih.gov/pubmed/15592008

60. Finnigan S, van Putten MJAM. EEG in ischaemic stroke: Quantitative EEG can uniquely inform (sub-)acute prognoses and clinical management. Clin Neurophysiol [Internet]. 2013;124(1):10-9. Available from:

http://dx.doi.org/10.1016/j.clinph.2012.07.003 


\section{APÊNDICE A ñ TERMO DE CONSENTIMENTO TERMO DE CONSENTIMENTO LIVRE E ESCLARECIDO}

\begin{tabular}{llcc} 
Eu,_, portador do documento de identificação/número: \\
\hline endereço:
\end{tabular}

telefone de contato: estou sendo convidado a participar de um estudo denominado óACHADOS ELETROENCEFALOGRÁFICOS EM PACIENTES PORTADORES DE ACIDENTE VASCULAR CEREBRALô, cujos objetivos são: avaliar as alterações no eletroencefalograma e estudar a relação com os sinais e sintomas dos pacientes vítimas de acidente vascular cerebral (derrame cerebral), bem como investigar achados que indiquem o risco do surgimento de crises epilépticas nestes pacientes.

A minha participação no referido estudo será no sentido de responder perguntas sobre a história da minha doença atual, relatar sintomas e o tempo e forma em que começaram a acontecer, além de doenças preexistentes. Também serei examinado pelo médico neurologista e por residentes da neurologia e farei o exame de eletroencefalografia (eletro da cabeça) durante a minha internação no Hospital de Base do Distrito Federal.

Fui alertado de que, da pesquisa a se realizar, posso esperar alguns benefícios, tais como: não ser o meu atendimento e tratamento neurológico atrasado ou interrompido por causa dos testes da pesquisa, além de ser também avaliado pelos médicos do projeto de pesquisa e ainda realizar o exame eletroencefalograma, o qual pode trazer informações importantes sobre meu estado de saúde e ajudar no tratamento realizado durante a minha internação.

Recebi, por outro lado, os esclarecimentos necessários sobre os possíveis desconfortos e riscos decorrentes do estudo, levando-se em conta que é uma pesquisa, e os resultados positivos ou negativos somente serão obtidos após a sua realização. Fui também informado de que para a realização do exame eu poderei ter que ser transportado dentro do próprio Hospital de Base até o setor onde o mesmo é realizado e aguardar com certa imobilidade e cooperação com as orientações das técnicas, por cerca de 45 a 60 minutos até que o exame seja concluído.

Estou ciente de que minha privacidade será respeitada, ou seja, meu nome ou qualquer outro dado ou elemento que possa, de qualquer forma, me identificar, será mantido em sigilo. 
Também fui informado de que posso me recusar a participar do estudo, ou retirar meu consentimento a qualquer momento, sem precisar justificar, e de, por desejar sair da pesquisa, não sofrerei qualquer prejuízo à assistência que venho recebendo.

Os pesquisadores envolvidos com o referido projeto são Pierre da Silva Paz Junior, Rafael Lourenço Duarte e Iruena Moraes Kessler e com eles poderei manter contato pelo telefone 61-83234433.

É assegurada a assistência durante toda pesquisa, bem como me é garantido o livre acesso a todas as informações e esclarecimentos adicionais sobre o estudo e suas conseqüências, enfim, tudo o que eu queira saber antes, durante e depois da minha participação.

Enfim, tendo sido orientado quanto ao teor de todo o aqui mencionado e compreendido a natureza e o objetivo do já referido estudo, manifesto meu livre consentimento em participar, estando totalmente ciente de que não há nenhum valor econômico, a receber ou a pagar, por minha participação.

Em caso de reclamação ou qualquer tipo de denúncia sobre este estudo devo ligar para o CEP FEPEX (61)3325-4964 OU 3326-0119 ou mandar um email para comitedeetica.secretaria@gmail.com

Brasília, de de 201 .

Nome e assinatura do paciente ou responsável

Pierre da Silva Paz Junior

Neurologista CRM-DF 19983

Pesquisador responsável 


\section{APÊNDICE B ñ FICHA DE COLETA DE DADOS CLÍNICOS}

\section{PROTOCOLO DE PESQUISA}

Nome:

Data Nascimento:

SES:

Data de admissäo:

- Data do ictus: $I_{1}{ }_{1}$.

- Tipo de AVE (isquêmico/Hemorrágico):

- Trombólise:

- Comorbidades/medicaçőes:

- Crise epiléptica (sim/não, tipo?):

Exame Clínico 1:

- NIHSS (total, -pontos em)

- Escala de Rankin Modificada:

- História h. (dia e hora)

- Exame físico (alterações)

- Etiologia (sim/näo? Qual?)

Exame Clínico 2: __ ________ h. (dia e hora)

- NIHSS (total, -pontos em):

- Escala de Rankin Modificada:

- História Clínica:

- Exame físico (alterações)

- Etiologia (sim/não? Qual?)

\section{Neuroimagem 1 :}

- Localização (frontal, occipital,...):

- Profundidade (córtico/subcortical):

- Volume (axbxc/2):

- Observaçöes (transformação/efeito de massa/hidrocefalia/hsa):

Neuroimagem 2:

- Localização (frontal, occipital,...)

- Profundidade (córtico/subcortical):

- Volume (axbxc/2):

- Observaçöes (transformação/efeito de massa/hidrocefalia/hsa):

\section{Dados da trombólise/Craniectomia}

- Delta ictus-agulha

- Observaçöes:

- Craniectomia (sim/näo?) 


\section{APÊNDICE C ñ FICHA DE COLETA DE DADOS DO ELETROENCEFALOGRAMA}

Nome:

Idade:

Data do AVC:

Data do exame:

\begin{tabular}{|c|c|c|c|c|}
\hline Tempo: & & & & \\
\hline Condições técnicas & & atórias & & atórias \\
\hline Nivel de conciência & Vigília & Sono & Torpor & \\
\hline Atividade de base & $\begin{array}{l}\text { Simétria } \\
\text { Assimétrica }\end{array}$ & & $\begin{array}{l}\text { Organizada } \\
\text { Desorganiza }\end{array}$ & \\
\hline $\begin{array}{l}\text { Ritmo dominante } \\
\text { Posterior }\end{array}$ & $\begin{array}{l}\text { Presente } \\
\text { Ausente }\end{array}$ & & Frequencia= & \\
\hline Atividade beta anterior & Presente & & Ausente & \\
\hline Estágios do sono & N1 & N2 & N3 & \\
\hline Grafoelementos do sono & $\begin{array}{l}\text { Presente } \\
\text { Ausente }\end{array}$ & & $\begin{array}{l}\text { Incidência h } \\
\text { Incidência a }\end{array}$ & do esperado \\
\hline $\begin{array}{l}\text { Atividade lenta } \\
\text { intermitente }\end{array}$ & $\begin{array}{l}\text { Delta ( } \mathrm{Hz}) \\
\text { Teta }(\mathrm{Hz})\end{array}$ & $\begin{array}{l}\mathrm{HCD} \\
\mathrm{HCE}\end{array}$ & $\begin{array}{l}\text { Rara } \\
\text { Ocasional } \\
\text { Frequente } \\
\text { Abundante }\end{array}$ & $\begin{array}{l}\text { Frontal } \\
\text { Parietal } \\
\text { Temporal } \\
\text { Occipital }\end{array}$ \\
\hline Atividade Epileptiforme & $\begin{array}{l}\text { Ponta } \\
\text { Onda aguda } \\
\text { Ponta-onda } \\
\text { Poliponta }\end{array}$ & $\begin{array}{l}\text { HCD } \\
\text { HCE }\end{array}$ & $\begin{array}{l}\text { Rara } \\
\text { Ocasional } \\
\text { Frequente } \\
\text { Abundante }\end{array}$ & $\begin{array}{l}\text { Rara } \\
\text { Ocasional } \\
\text { Frequente } \\
\text { Abundante }\end{array}$ \\
\hline Atividade periódica & $\begin{array}{l}\text { Delta rítmica } \\
\text { intermitente }\end{array}$ & $\begin{array}{l}\text { Delta } \\
\text { polimórfica }\end{array}$ & & \\
\hline
\end{tabular}




\begin{tabular}{|l|l|l|l|l|}
\hline & monomórfica & (PDA) & & \\
\hline FEI & Fotodriving & $\begin{array}{l}\text { Resposta } \\
\text { fotomioclonica }\end{array}$ & $\begin{array}{l}\text { Resposta } \\
\text { fotoparoxística }\end{array}$ & \\
\hline Conclusão & Normal & Anormal & & \\
\hline
\end{tabular}




\section{APÊNDICE D - ARTIGO}

\section{Concordância Interobservador dos achados do EEG em pacientes na fase aguda e subaguda do Acidente Cerebrovascular Isquêmico}

Interobserver agreement of EEG in acute and subacute phases in a cerebrovascular disease

Pierre da Silva Paz Júnior ${ }^{1}$, Pedro Alessandro Leite De Oliveira ${ }^{2}$, André Gustavo Fonseca Ferreira ${ }^{3}$, Leonardo Petrus da Silva $\mathrm{Paz}^{4}$, Wagner Afonso Teixeira ${ }^{2}$, Iruena Moraes Kessler ${ }^{5}$

Paz Júnior $\mathrm{PS}^{1}$, Oliveira $\mathrm{PAL}^{2}$, Ferreira $\mathrm{AGF}^{3}, \mathrm{Paz} \mathrm{LPS}^{4}$, Teixeira $\mathrm{WA}^{2}$, Kessler $\mathrm{IM}^{5}$

${ }^{1}$ Médico neurologista, neurofisiologista, aluno de Mestrado do Programa de Ciências Médicas, UnB, Brasília - DF, Brasil.

${ }^{2}$ Médico, neurologista, neurofisiologista do Hospital de Base do Distrito Federal, Brasília - DF, Brasil.

${ }^{3}$ Médico, neurologista, neurofisiologista do Hospital de Base do Distrito Federal, Professor da Escola Superior de Ciências da Saúde (ESCS), Brasília - DF, Brasil.

${ }^{4}$ Professor Universidade de Brasília, Campus Ceilândia, Brasília - DF, Brasil.

${ }^{5}$ Professor Universidade de Brasília, Programa de Pós-Graduação em Ciências Médicas, Campus Darcy Ribeiro, Brasília - DF, Brasil.

\section{Resumo:}

OBJETIVO: Determinar a taxa de concordância interexaminador de pacientes atendidos em um hospital terciário do Distrito Federal com acidente vascular cerebral isquêmico (AVC) durante a fase aguda. MATERIAIS E MÉTODOS: Os 
eletroencefalogramas (EEG) de 18 pacientes com diagnóstico clínico e radiológico de AVC na fase aguda a subaguda, de ambos os sexos, com 21 a 77 anos, submetidos ou não à trombólise endovenosa foram avaliados por três eletroencefalografistas de forma independente. A taxa de concordância interexaminador foi calculada pelo teste kappa. RESULTADOS: O EEG foi laudado como anormal em $78 \%$ dos pacientes. A atividade lenta focal (delta ou teta) foi o achado anormal mais frequente, sendo intermitente em cerca de $33 \%$ da amostra e contínua em $28 \%$ destes. As taxas de concordância interexaminador encontradas foram substanciais para a simetria da atividade elétrica cerebral, presença de atividade lenta anormal, presença de atividade epileptiforme e para a conclusão do EEG (normal versus anormal). Os critérios organização da atividade de base e lateralização dos achados anormais pelo EEG apresentaram concordância apenas moderada. CONCLUSÃO: Neste estudo o EEG apresentou concordância interexaminador moderada a substancial, sendo necessária a implementação de medidas para aumentar a aplicabilidade clínica do método nos pacientes com AVC.

Palavras-chave: Eletroencefalografia, EEG, Acidente Vascular Cerebral, Concordância interexaminador.

\section{Abstract:}

OBJECTIVES: To determine the interrater concordance rate of patients treated in a tertiary hospital in the Distrito Federal, Brazil, with ischemic stroke (IS) during the acute phase. MATERIALS AND METHODS: The electroencephalogram (EEG) of 18 patients with clinical and radiological diagnosis of stroke in acute subacute, of both sexes, with 21 to 77 years, submitted or not to intravenous thrombolysis were 
evaluated by three EEG readers independently . The interrater agreement rate was calculated using the kappa test. RESULTS: The EEG was reported abnormal in $78 \%$ of patients. The focal activity slow (delta and theta) was the most common abnormal finding, intermittent being about $33 \%$ of the sample and continous in $28 \%$ thereof. The interrater agreement rates found were substantial for the symmetry of brain electrical activity, presence of abnormal slow activity, the presence of epileptiform activity and the completion of the EEG (normal versus abnormal). The criteria of EEG background activity`s organization and lateralization of abnormal findings by EEG showed only moderate agreement. CONCLUSION: In this study the EEG showed moderate or substancial interrater agreement, requiring the implementation of measures to increase the clinical applicability of the method in patients with stroke.

Key-words: Electroencephalography, EEG, stroke, Interobserver agreement, interratter agreement. 


\section{INTRODUÇÃO:}

As doenças cerebrovasculares constituem a terceira maior causa de mortalidade e a principal causa de incapacidade no mundo ${ }^{1}$. Apesar da tendência de queda na mortalidade nos últimos anos, o Brasil, entre todos os países da América Latina, é o que ainda apresenta as maiores taxas de mortalidade por acidente vascular cerebral $(A \vee C)^{2}$. Em 2005 o acidente vascular cerebral isquêmico (AVCi) isoladamente foi responsável por $10 \%$ de todas as mortes (90006 mortes) e 10\% de todas as admissões em hospitais públicos, sendo a principal causa de morte e incapacidade no Brasil ${ }^{3}$.

A utilidade clínica do eletroencefalograma (EEG) tem sido amplamente discutida através das últimas sete décadas. Uma revisão ampla de tais estudos propôs que o EEG tem utilidade no diagnóstico precoce, avaliação prognóstica, seleção de pacientes para tratamento, no manejo clínico e na deteç̧ão de crises em pacientes com AVC isquêmico agudo ${ }^{59}$.

Sobre as alterações eletroencefalográficas relacionadas à doença cerebrovascular, foram descritos achados relacionados a quadros de maior ou menor gravidade clínica. A lentificação difusa ou focal na faixa delta e a deterioração dos ritmos rápidos da atividade elétrica cerebral de base indicam um pior prognóstico, com curso mais maligno ${ }^{19}$. Em oposição, a preservação do ritmo cerebral de fundo rápido, a ausência de atividade delta e a presença de atividade teta ou mais rápida no foco são preditores de um curso mais benigno ${ }^{18,19}$.

Estudos recentes tem utilizado o EEG quantitativo (QEEG) com o objetivo de acrescentar informações e tornar menos subjetiva a avaliação destes pacientes. 
Entre os índices utilizados estão a quantificação do poder da atividade lenta anormal em relação àquela mais rápida e a avaliação da assimetria de voltagem interhemisférica ${ }^{60}$.

Apesar da introdução de padronizações da técnica de aquisição, leitura e nomenclatura, a interpretação do EEG permanece sendo subjetiva, variando de acordo com as características individuais dos eletroencefalografistas o que pode afetar as taxas de concordância interobservador ${ }^{51}$.

O presente estudo visa avaliar o perfil eletroencefalográfico de pacientes vítimas de acidente vascular cerebral isquêmico durante a fase aguda atendidos em um hospital terciário do Distrito Federal bem como avaliar o grau de concordância interobservador dos registros realizados.

\section{MATERIAS E MÉTODOS}

Trata-se de um estudo observacional, analítico, transversal, destinado a avaliação do grau de concordância interobservador. A presente pesquisa foi aprovada pelo parecer com número CAAE FEPECS 33843314.2.0000.5553 e do comitê de ética local do Hospital de Base do Distrito Federal. Todos os pacientes preencheram o termo de consentimento livre e esclarecido.

Foram admitidos no estudo os pacientes maiores de 18 anos, com quadro inédito de Acidente Vascular Cerebral Isquêmico em fase aguda em um hospital público terciário da cidade de Brasília-DF, Brasil, no período de outubro de 2014 a julho de 2015, submetidos a avaliação pela equipe de neurologistas daquele hospital 
e que tivessem realizado ao menos um exame de neuroimagem (tomografia de crânio não contrastada de 32 canais ou ressonância magnética de crânio 1,5 T), submetidos ou não ao procedimento de trombólise venosa com alteplase (r-TPA) e que tiveram ao menos um registro eletroencefalográfico considerado tecnicamente satisfatório realizado até sete dias após o início dos déficits. As informações sobre os dados clínicos e de neuroimagem dos pacientes foram obtidos do prontuário eletrônico em revisão.

Foram excluídos os pacientes com histórico de abordagem neurocirúrgica, epilepsia pré-existente, diagnóstico clínico, laboratorial e ou radiológico de traumatismo cranioencefálico, hemorragia subaracnóidea espontânea, trombose venosa cerebral, distúrbios hidroeletrolíticos ou metabólicos graves que não preencheram critérios para doença cerebrovascular arterial como causa primária do atendimento, condições patológicas neurodegenerativas, história de acidentes vasculares cerebrais prévios ou de outras comorbidades que pudessem alterar a acurácia das medidas realizadas.

O eletroencefalograma (EEG) foi realizado com aparelho de 21 canais, com eletrodos colocados de acordo com o sistema internacional 10-20, analisado pelas montagens bipolares e referenciais comuns, sob banda de filtro de 1,0 a $70,0 \mathrm{~Hz}$ e com sensibilidade de 7 microvolts por milímetro, conforme recomendações da Academia Americana de Neurofisiologia Clínica/Sociedade Brasileira de Neurofisiologia Clínica (SBNC) $)^{52}$.

Os registros foram interpretados por meio de análise visual e independente. Os casos foram distribuídos de forma aleatória entre dois ou três eletroencefalografistas experientes com titulação em neurofisiologia clínica. Os 
avaliadores não possuiam conhecimento sobre a história clínica, gravidade do quadro ou se os pacientes haviam sido submetidos ou não à trombólise endovenosa.

A análise dos traçados foi orientada por um formulário padronizado contendo as seguintes questões: qualidade técnica de gravação, organização e simetria da atividade elétrica cerebral de base, presença e tipo de atividade lenta (contínua versus intermitente), presença de atividade epileptiforme, lateralização dos achados anormais e conclusão do exame (normal versus anormal).

A análise estatística descritiva e a análise de concordância pelo teste kappa foram realizadas com o auxílio do software IBM SPSS Statistics ${ }^{\circledR}$ versão 22. Foram consideradas as seguintes faixas de valores para classificação do grau de concordância pelo coeficiente de kappa: valores $\leq 0$ indicando ausência de concordância, $0.01-0.20$ como nenhuma a leve concordância, $0.21-0.40$ como pobre, $0.41-0.60$ como moderada, $0.61-0.80$ como substancial e $0.81-1.00$ como quase perfeita ${ }^{53}$.

As variáveis da pesquisa utilizadas para análise de concordância foram: assimetria da atividade de base, organização da atividade de base, presença de atividade lenta, presença de atividade epileptiforme, achados anormais lateralizatórios e conclusão do exame. 


\section{RESULTADOS}

Foram avaliados 44 pacientes, dos quais quatro foram excluídos por apresentarem história pregressa de doença cerebrovascular, um paciente por ter apresentado AVC primariamente hemorrágico, sete pacientes por terem a qualidade de seus registros eletroencefalográficos considerada insatisfatória por um ou mais dos três eletroencefalografistas e seis por terem laudo de apenas um dos examinadores utilizados para análise comparativa. A aplicação dos critérios de exclusão resultou na seleção de 18 pacientes.

Para análise descritiva da amostra foram utilizados os laudos dos pacientes realizados pelo examinador 1 , o qual foi escolhido arbitrariamente pelo critério de ter laudado o maior número de exames, considerando a amostra inicial dos quarenta e quatro pacientes. Para a análise comparativa entre observadores foram utilizados os dados dos examinadores 1 e 2 devido ao maior número de laudos concomitantemente obtidos destes examinadores.

$\mathrm{Na}$ tabela 1 estão descritas as características demográficas, clínicas e os achados de neuroimagem da amostra. Os pacientes apresentaram média de idade de 50 anos, sendo que a maioria não foi submetida à trombólise endovenosa. Nenhum paciente teve crises epilépticas clinicamente detectadas até o momento da realização do eletroencefalograma.

Todos os pacientes apresentaram lesões detectáveis à tomografia computadorizada de crânio (TCC) ou à Ressonância Magnética de Crânio. Três pacientes apresentaram TCC normais na admissão sendo submetidos a um novo exame de neuroimagem confirmatório durante a internação. As lesões identificadas 
foram classificadas quanto à sua lateralização, não sendo, entretanto, classificadas quanto à topografia ou profundidade.

A análise dos dados do EEG revelou que o intervalo de tempo entre o ictus vascular e a realização do eletroencefalograma variou de 0 a 7 dias com média de 3,9 dias. A duração da gravação dos registros variou de 21 a 41 minutos com média de 33,8 minutos. À partir da análise dos laudos realizados pelo Examinador 1, obtivemos os resultados descritos a seguir.

Cerca de vinte e oito por cento dos pacientes apresentaram uma atividade elétrica cerebral assimétrica, ou seja, traçados que apresentaram uma diferença significativa de amplitude ou de frequência dos ritmos da atividade elétrica cerebral. Metade dos pacientes apresentou critérios para a desorganização da atividade elétrica cerebral de base. Foram classificados como tendo atividade elétrica cerebral de base desorganizada os exames que preencheram ao menos um dos seguintes critérios: lentificação do ritmo posterior (menor ou igual a $8,0 \mathrm{~Hz}$ ), ausência ou redução da presença dos ritmos rápidos em regiões anteriores ou redução da amplitude dos ritmos elétricos cerebrais (menor que $10 \mathrm{mcV} / \mathrm{mm}$ ).

Foi observada a presença de atividade lenta anormal na faixa delta e ou teta em cerca de $61 \%$ dos pacientes. Destes, pouco mais da metade (54\%) apresentaram atividade lenta focal intermitente e os demais apresentaram atividade lenta anormal contínua. Apenas $39 \%$ dos pacientes não apresentaram regiões de lentificação focal em seus traçados.

Dos 18 pacientes analisados, apenas dois apresentaram grafoelementos epileptiformes (ondas agudas e espículas) em seus traçados. Entretanto, não foi relatada atividade epiléptica clínica pelos médicos assistentes em nenhum dos 
pacientes. Anormalidades unilaterais, ou seja, achados lateralizatórios, foram percebidos em $61 \%$ dos pacientes, enquanto o restante apresentou achados bilaterais ou exames normais.

O eletroencefalograma foi laudado como anormal em cerca de $78 \%$ dos pacientes estudados. Nestes, houve a presença de ao menos um dos seguintes critérios: presença de desorganização ou assimetria da atividade elétrica cerebral de base, presença de atividade lenta focal ou generalizada, presença de grafoelementos epileptiformes e a presença de outros grafoelementos anormais. A caracterização dos achados eletroencefalográficos realizada pelo examinador 1 encontra-se resumida na tabela 2.

À partir dos dados obtidos da avaliação dos traçados pelos examinadores $1 \mathrm{e}$ 2 foi realizada a avaliação do nível de concordância interexaminadores pelo teste kappa. Foram obtidos os seguintes resultados: a simetria da atividade elétrica cerebral de base, a presença de atividade lenta anormal (não especificada como contínua ou intermitente), a presença de atividade epileptiforme e a conclusão sobre a presença de qualquer anormalidade apresentaram concordância substancial entre os examinadores 1 e 2. A avaliação da organização da atividade de base e da lateralização dos achados anormais pelo EEG apresentaram concordância apenas moderada. A tabela 3 mostra os resultados das análises dos observadores 1 e 2 e a tabela 4 resume os achados da análise da concordância interexaminador nesta amostra. 


\section{DISCUSSÃO:}

No presente trabalho foi avaliada a concordância interexaminador do EEG em pacientes portadores de doença cerebrovascular e foi traçado o perfil dos pacientes de um hospital terciário do Distrito Federal. Este foi o primeiro estudo a comparar a taxa de concordância interobservador em pacientes portadores de AVC.

Os sinais de EEG são complexos e a inspeção visual dos traçados para extrair características relevantes é sujeita a variabilidade individual ${ }^{54}$. Estudos prévios observaram uma taxa de concordância interexaminador variando amplamente de $19 \%{ }^{55}$ a $95 \%{ }^{56}$.

Embora não exista uma padronização internacional quanto aos critérios e classificação dos achados eletroencefalográficos, foi observada no presente estudo uma substancial concordância interexaminador para a maior parte dos critérios analisados. Isso pode ter ocorrido devido ao uso de categorias mais amplas de classificação, o que segundo estudos prévios $^{57}$ pode aumentar o grau de concordância quando comparada a categorias mais específicas como a presença de atividade epileptiforme.

Variações nas características relatadas dos testes podem ser relacionadas a diferenças nas características dos pacientes ou aos limiares dos examinadores ao interpretar os testes como positivos, sendo no último aspecto relacionado a variação de até $33 \%$ da acurácia do exame ${ }^{58}$. 
Concordamos com as recomendações de Gerber que propõe medidas para o aumento da concordância interobservador na avaliação do EEG, tais como (1) manter a simplicidade da terminologia, (2) limitar o uso de descritores opcionais, (3) aumentar as definições publicadas com exemplos visuais que ilustram os limites de um termo individual adicionalmente aos protótipos, (4) desenvolver um treinamento interativo incluindo o teste dos participantes que participarão da pesquisa.

O trabalho possui algumas limitações tais como: o tamanho da amostra, o uso de amostragem não probabilística, a ausência de avaliação eletroencefalográfica quantitativa e a ausência de localização acurada das lesões (distinção entre lesões corticais e subcorticais e sua topografia) à neuroimagem.

Recomenda-se a padronização da terminologia para aumentar o nível de concordância interexaminador, a confiabilidade e para maximizar a comunicação entre os diferentes revisores de eletroencefalogramas de um mesmo paciente ${ }^{54}$.

Os dados deste estudo sugerem uma substancial concordância interexaminador em relação a alguns ítens da avaliação, tais como assimetria da atividade de base, presença de atividade lenta, presença de atividade epileptiforme e conclusão do exame, entretanto são necessários novos estudos utilizando terminologia e critérios de classificação para aumentar a concordância interexaminador

\section{REFERÊNCIAS}

1. Marsh JD, Keyrouz SG. Stroke prevention and treatment. J Am Coll Cardiol. 2010;56(9):683-91. 
2. Lotufo P a. Stroke: a neglected disease in Brazil. São Paulo Med J. 2005;123(1):3-4.

3. Martins SCO, Freitas GR De, Pontes-Neto OMOM, Pieri A, Moro CHC, Jesus PAP De, et al. Guidelines for acute ischemic stroke treatment: part I. Arq Neuropsiquiatr [Internet]. 2012;70(11):885-93. Available from: http://ovidsp.ovid.com/ovidweb.cgi?T=JS\&PAGE=reference\&D=psyc7\&NEWS $=\mathrm{N} \& \mathrm{AN}=2012-23551-$

012\%5Cnhttp://www.ncbi.nlm.nih.gov/pubmed/23175203\%5Cnhttp://www.ncbi. nlm.nih.gov/pubmed/22899035

4. Baldin CF, Alexandrov A V, Bellavance A, Bornstein N, Chambers B, Coté R, et al. Seizures after stroke. Arch neurol [Internet]. 2000;57:1617-22. Available from: http://www.ncbi.nlm.nih.gov/pubmed/11074794

5. Chen T-C, Chen Y-Y, Cheng P-Y, Lai C-H. The incidence rate of post-stroke epilepsy: a 5-year follow-up study in Taiwan. Epilepsy Res [Internet].

2012;102(3):188-94. Available from:

http://www.ncbi.nlm.nih.gov/pubmed/22749919

6. Romaniak A B-GM. Post-Stroke Epilepsy. Neurol Neurochir Pol. 1998;MayJun(32(3)):603-13.

7. Burn J, Dennis M, Bamford J, Sandercock P, Wade D, Warlow C. Epileptic seizures after a first stroke: the Oxfordshire Community Stroke Project. BMJ [Internet]. 1997;315(7122):1582-7. Available from:

http://www.pubmedcentral.nih.gov/articlerender.fcgi?artid=2127973\&tool=pmce ntrez\&rendertype=abstract

8. Hauser WA. Seizure disorders. Epilepsia. 1992;33(suppl 4:S6-14.

9. Luhdorf K, Jensen LK PA. Etiology of seizures in the elderly. Epilepsia. 1986;27:458-63.

10. Olsen T. Post-stroke epilepsy. Curr Arthroscler Rep. 2001;3:340-4.

11. Niedermeyer, E., Silva F.L., Tettenborn B. Cerebrovascular Diseases and EEG. In: Electroencephalography, Basic Principles, Clinical Applications, and Related Fields Williams and Wilkins, Philadelphia,PA, Sixth Edition. 2011. p. 351-74.

12. Claassen J, Hirsch LJ, Kreiter KT, Du EY, Connolly ES, Emerson RG MS. Quantitative continuous EEG for detecting delayed cerebral ischemia in patients with poor-grade subarachnoid hemorrhage. Clin Neurophysiol. 2004;Dec(115(12)):2699-710.

13. Hossmann K. Viability thresholds and the penumbra of focal ischemia. Ann Neurol. 1994;36:557-65.

14. Genvins A, Le J, Brickett $P$ et al. Seeing through the skull: advanced EEG use MRIs to accurately measure cortical activity from the scalp. Brain Topogr. 1991;4:125-31. 
15. Jackel, RA, Harner R. Computed EEG topography in acute stroke. Neurophysiol Clin. 1989;19:185-97.

16. Luu P, Tucker DM, Englander $R$ et al. Localizing acute stroke-related EEG changes: assessing the effects of spatial undersampling. J Clin Neurophysiol. 2001;18:302-17.

17. Ebersole, J. S., Pedley TA. Current practice of clinical electroencephalography. Philadelphia: Lippincott Williams \& Wilkins, 3a Edição, 2003. Capítulo 11. 304$11 \mathrm{p}$.

18. Cillessen JP, van Huffelen a C, Kappelle LJ, Algra A, van Gijn J.

Electroencephalography improves the prediction of functional outcome in the acute stage of cerebral ischemia. Stroke [Internet]. 1994 Oct;25(10):1968-72. Available from: http://www.ncbi.nlm.nih.gov/pubmed/8091439

19. Burghaus L, Hilker R, Dohmen C, Bosche B, Winhuisen L, Galldiks N, et al. Early electroencephalography in acute ischemic stroke: Prediction of a malignant course? Clin Neurol Neurosurg. 2007;109(1):45-9.

20. Collice M, Arena O, Fontana RA et al. Role of EEG monitoring and crossclamping duration in carotid endarterectomy. J Neurosurg. 1986;65:815-9.

21. Holmes $\mathrm{G}$. The electroencephalogram as a predictor of seizures following cerebral infarction. Clin Electroencephalogr. 1980;11:83-6.

22. Kayser-Gatchalian MC NB. The prognostic value of EEG in ischaemic cerebral insults. Electroencephalogr Clin Neurophysiol. 1980;49:608-17.

23. Van der Drift JHA MO. The EEG in cerebral ischemic lesions. Correlations with clinical and pathological findings. In: In: Gastaut H, Meyer JS, eds Cerebral Annoxia and the Electroencephalogram Springfield IL: Thomas; 1961. p. 18096.

24. Van der Drift J.H.A. KNKD. The EEG in cerebrovascular disorders in relations to pathology. In: In: A REmond, Ed-in-chief Handbook of Electroencephalography and Clinical Neurophysiology Vol 14A Amsterdan: Elsevier; 1972. p. 12-130, 47-64.

25. Pohlmann-Eden B, Hoch DB CJ. Periodic Lateralized epileptiform discharges: a critical rewiew. JClin Neurophysiol. 1996;13:519-30.

26. Sainio K, Stenberg D KI. Visual and spectral EEG analysis in the evaluation of the outcome in patients with ischemic brain infarction. Electroencephalogr Clin Neurophysiol. 1983;56:117-24.

27. Joynt, RJ; Cape, CA; Knott J. Significance of focal delta activity in adult electroencephalogram. Arch Neurol. 1965;12:631-638.

28. Hirsch LJ, LaRoche SM, Gaspard N, Gerard E, Svoronos A, Herman ST, et al. American Clinical Neurophysiology Society's Standardized Critical Care EEG Terminology: 2012 version. J Clin Neurophysiol. 2013;30(1):1-27.

29. Gaches J. Les activtiés périodiques en EEG. Rev EEG Neurophysiol. 
1971;1:9-33.

30. Fitzpatrick, W, Lowry N. PLEDS: clinical correlates. Can J Neurol Sci. 2007;34:443-50.

31. Müller C, Ackermann P BM. Visual and spectral analysis of sleep EEG in acute hemispheric stroke. Eur Neurol. 2002;48(164-171).

32. Gottselig JM, Bassetti CL AP. Power and coherence of sleep spindle frequency activity following hemispheric stroke. Brain. 2002;125:373-83.

33. Jasper $\mathrm{H}$, Van Buren J. Interrelationships between cortex and subcortical structures: clinical electroencephalographic studies. Electroencephalogr Clin Neurophysiol Suppl. 1953;4:168-88.

34. Giubilei $F$, lannilli $M$, Vitale A. Sleep patterns in acute ischemic stroke. Acta Neurol Scand. 1992;86:567-71.

35. Vock J, Ackermann P, Bischof M. Evolution of sleep and sleep EEG after hemispheric stroke. Brain. 2002;125:373-83.

36. Engel $\mathrm{S}$, Lechner $\mathrm{H}$, Logar $\mathrm{C}$. Clinical value of EEG in transient ischemic attacks. In: In: Lechner H, Aranibar A, eds EEG and clinical neurophysiology Amsterdam: Excerpta Medica. 1980. p. 173-80.

37. Madkour $\mathrm{O}$, Elwan $\mathrm{O}$, Hamdy $\mathrm{H}$. Transient ischemic attacks: electrophysiological (conventional and topographic EEG) and radiological (CCT) evaluation. J Neurol Sci. 1993;119:8-17.

38. Marshall D, Brey R, Morse M. Focal and/or lateralized polymorphic delta activity: association with either anormal or nonfocal computed tomographic scans. Arch Neurol. 1998;45:33-5.

39. Bruens J, Gastaut H, Gioive G. Electroencephalographic study of the signs of chronic vascular insufficiency of the Sylvian region in aged people.

Electroencephalogr Clin Neurophysiol. 1960;12:283-95.

40. Gastaut H, Bruens J, Roger J. Etude electroencephalographique des signes d'insuffisiance circulatoire syvienne chronique. Rev Neurol. 1959;100:59-65.

41. Markand O. EEG in the locked-in syndrome. Electroencephalogr Clin Neurophysiol. 1976;40:529-34.

42. Schaul N, Gloor P, Gotman J. The EEG in deep midline lesions. Neurology. 1981;31:157-67.

43. Westmoreland B, Klass D, Sharbrough F. Alpha coma: electroencephalographic, clinical, pathological and etiological correlation. Arch Neurol. 1998;32:713-8.

44. Macdonell R, Donnan G, Bladin P. The electroencephalogram and acute ischemic stroke. Arch Neurol. 1988;45:520-524.

45. Petty G, Labar D, Fisch B. Electroencephalography in lacunar infarction. J 
Neurol Sci. 1995;134:47-50.

46. Pohlmann-Eden B, Hoch O, Cochius J. Stroke and epilepsy: critical review of the literature. Part I: Epidemiology and risk factors. Cerebrovasc Dis. 1996;6:332-8.

47. Nagata K, Mizukami M, Araki G. Topographic electroencephalographic study of cerebral infarction using computed mapping of the EEG. J Cereb Blood Flow Metab. 1982;2:79-88.

48. Nagata K, Yunoki K, Araki G. Topographic electroencephalographic study of transient ischemic attacks. Electroencephalogr Clin Neurophysiol. 1984;58:291-301.

49. Nuwer M, Jordan S, Ahn S. Evaluation of stroke using EEG frequency analysis and topographic mapping. Neurology. 1987;37:1153-9.

50. Sheorajpanday RVA, Nagels G, Weeren AJTM, De Deyn PP. Quantitative EEG in ischemic stroke: Correlation with infarct volume and functional status in posterior circulation and lacunar syndromes. Clin Neurophysiol [Internet]. 2011;122(5):884-90. Available from: http://dx.doi.org/10.1016/j.clinph.2010.08.020

51. Gerber PA, Chapman KE, Chung SS, Drees C, Maganti RK, Ng Y-T, et al. Interobserver agreement in the interpretation of EEG patterns in critically ill adults. J Clin Neurophysiol. 2008;25(5):241-9.

52. Nuwer MR, Comi C, Emerson R, Fuglsang-Frederiksen a., Gu'erit JM, Hinrichs $\mathrm{H}$, et al. IFCN standards for digitial recording of clinical EEG.

Electroencephalogr Clin Neurophysiol. 1998;106:259-61.

53. McHugh M. Interrater reliability: the kappa statistic. Biochem Medica [Internet]. 2012;22(3):276-82. Available from:

http://hrcak.srce.hr/index.php?show=clanak\&id_clanak_jezik=132393

54. Tatum WO, Olga S, Ochoa JG, Munger Clary H, Cheek J, Drislane F, et al. American Clinical Neurophysiology Society Guideline 7: Guidelines for EEG Reporting. J Clin Neurophysiol [Internet]. 2016;33(4):328-32. Available from: http://content.wkhealth.com/linkback/openurl?sid=WKPTLP:landingpage\&an=0 0004691-20160800000009\%5Cnhttp://www.ncbi.nlm.nih.gov/pubmed/27482790

55. Williams GW, Lüders HO, Brickner A, Goormastic M, Klass DW. Interobserver variability in EEG interpretation. Neurology [Internet]. 1985 Dec;35(12):1714-9. Available from: http://www.ncbi.nlm.nih.gov/pubmed/4069362

56. Struve FA, Dorothy I, Green MA, Howard A. Reliability of Clinical Interpretation of the Electroencephalogram. 1975;6(2):54-60.

57. Gilbert DL, Sethuraman G, Kotagal U, Buncher CR. Meta-analysis of EEG test performance shows wide variation among studies. Neurology. 2002;60:564-70.

58. Deeks J. Systematic reviews of evaluations of diagnostic and screening tests. 
Bmj [Internet]. 2001;323(7305):157-62. Available from: http://www.bmj.com/content/323/7305/157.1.full

59. Jordan KG. Emergency EEG and continuous EEG monitoring in acute ischemic stroke. J Clin Neurophysiol [Internet]. 2004;21(5):341-52. Available from: http://www.ncbi.nlm.nih.gov/pubmed/15592008

60. Finnigan S, van Putten MJAM. EEG in ischaemic stroke: Quantitative EEG can uniquely inform (sub-)acute prognoses and clinical management. Clin Neurophysiol [Internet]. 2013;124(1):10-9. Available from: http://dx.doi.org/10.1016/j.clinph.2012.07.003

Tabela 1. Características clínicas e demográficas da amostra.

\begin{tabular}{llll}
\hline Característica & Categoria & $\mathrm{n}$ & $\%$ \\
\hline Idade & Mínima & 21 & \\
& Máxima & 77 & \\
& Média & 50 & \\
Sexo & Feminino & 9 & $50 \%$ \\
Trombólise Endovenosa & Masculino & 9 & $50 \%$ \\
& Sim & 5 & $28 \%$ \\
Craniectomia & Não & 13 & $72 \%$ \\
Crise Epiléptica & Sim & 1 & $6 \%$ \\
& Não & 17 & $94 \%$ \\
Lesão à Neuroimagem+ & Sim & 0 & $0 \%$ \\
& Não & 18 & $100 \%$ \\
Lateralização à neuroimagem & Sim & 18 & $100 \%$ \\
& Não & 0 & $0 \%$ \\
& Esquerda & 11 & $61 \%$ \\
& Direita & 6 & $33 \%$ \\
\hline
\end{tabular}

Legenda: $E E G=$ eletroencefalograma; Delta Ictus-EEG $=$ intervalo de tempo entre início do quadro isquêmico até a realização do EEG; += Tomografia computadorizada de crânio e ou Ressonância Magnética de Crânio.

Fonte: Dados da pesquisa 2015. 


\begin{tabular}{llll}
\hline Tabela 2. Características Eletroencefalográficas da amostra*. & & \\
\hline & & \multicolumn{2}{l}{ Examinador 1} \\
Característica & Categoria & $\mathrm{n}$ & $\%$ \\
\hline Delta Ictus-EEG (em dias) & Mínimo & 0 & \\
& Máximo & 7 & \\
& Média & 3,9 & \\
Duração do EEG (em minutos) & Mínima & 21 \\
& Máxima & 41 & \\
Simctria da atividade de basc & Média & 33,8 & \\
& Simćtrica & 13 & $72 \%$ \\
Organização da atividade de base & Assimétrica & 5 & $28 \%$ \\
Presença de atividade lenta anormal & Organizada & 9 & $50 \%$ \\
Atividade lenta anormal (tipo) & Desorganizada & 9 & $50 \%$ \\
& Sim & 7 & $39 \%$ \\
& Não & 11 & $61 \%$ \\
Atividade epileptiforme & Ausente & 7 & $39 \%$ \\
& Intermitente & 6 & $33 \%$ \\
Lateralização & Contínua & 5 & $28 \%$ \\
& Sim & 2 & $11 \%$ \\
Conclusão do exame & Não & 16 & $89 \%$ \\
& Direita & 3 & $17 \%$ \\
& Esquerda & 8 & $44 \%$ \\
& Não lateralizada & 7 & $39 \%$ \\
& Normal & 4 & $22 \%$ \\
& Anormal & 14 & $78 \%$ \\
\hline
\end{tabular}

Legenda:*Dados da análise do Examinador 1; EEG=eletroencefalograma; Delta Ictus-EEG= intervalo de tempo entre início do quadro isquêmico até a realização do EEG;

Fonte: Dados da pesquisa 2015. 


\begin{tabular}{|c|c|c|c|c|c|}
\hline \multirow[b]{2}{*}{ Característica } & \multirow[b]{2}{*}{ Categoria } & \multicolumn{2}{|c|}{ Examinador 1} & \multicolumn{2}{|c|}{ Examinador 2} \\
\hline & & $\mathrm{n}$ & $\%$ & $\mathrm{n}$ & $\%$ \\
\hline \multirow[t]{2}{*}{ Simétrica } & Sim & 13 & $72 \%$ & 11 & $61 \%$ \\
\hline & $\mathrm{NaO}$ & 5 & $28 \%$ & 7 & $39 \%$ \\
\hline \multirow[t]{2}{*}{ Organizada } & Sim & 9 & $50 \%$ & 11 & $61 \%$ \\
\hline & Não & 9 & $50 \%$ & 7 & $39 \%$ \\
\hline \multirow[t]{2}{*}{ Atividade lenta anormal } & Sim & 11 & $61 \%$ & 6 & $33 \%$ \\
\hline & Não & 7 & $39 \%$ & 12 & $67 \%$ \\
\hline \multirow[t]{3}{*}{ Atividade lenta anormal (tipo) } & Ausente & 7 & $39 \%$ & 6 & $33 \%$ \\
\hline & Intermitente & 6 & $33 \%$ & 5 & $28 \%$ \\
\hline & Contínua & 5 & $28 \%$ & 7 & $39 \%$ \\
\hline \multirow[t]{2}{*}{ Atividade epileptiforme } & Sim & 2 & $11 \%$ & 1 & $6 \%$ \\
\hline & Não & 16 & $89 \%$ & 17 & $94 \%$ \\
\hline \multirow[t]{3}{*}{ Lateralização } & Direita & 3 & $17 \%$ & 3 & $17 \%$ \\
\hline & Esquerda & 8 & $44 \%$ & 9 & $50 \%$ \\
\hline & Não lateralizada* & 7 & $39 \%$ & 6 & $33 \%$ \\
\hline \multirow[t]{2}{*}{ Conclusão do exame } & Normal & 4 & $22 \%$ & 4 & $22 \%$ \\
\hline & Anormal & 14 & $78 \%$ & 14 & $78 \%$ \\
\hline
\end{tabular}

Legenda: $\mathrm{EEG}=$ eletroencefalograma, * =EEG normal ou anormalidades bilaterais.

Fonte: Dados da pesquisa 2015.

\begin{tabular}{|c|c|c|c|}
\hline Critério Analisado & Nível de Concordância E1 x E2 & Kappa & Significância \\
\hline Assimetria da Atividade de Base & Substancial & 0,753 & $p=0,001$ \\
\hline Organização da Atividade de Base & Moderada & 0,556 & $p=0,016$ \\
\hline Presença de Atividade Lenta & Substancial & 0,668 & $p=0,00$ \\
\hline Presença de Atividade Epileptiforme & Substancial & 0,64 & $p=0,004$ \\
\hline Achados lateralizatórios & Moderada & 0,463 & $p=0,008$ \\
\hline Conclusão do Exame & substancial & 0,679 & $p=0,004$ \\
\hline
\end{tabular}

Legenda: E1= Examinador 1; E2=Examinador; Kappa=Teste de Concordância de Kappa; $p=p$ valor. 


\section{ANEXO A ñ TERMO DE CONCORDÂNCIA DE PESQUISA}

\section{TERMO DE CONCORDÂNCIA}

O Dr. André Gustavo Fonseca Ferreira, chefe da Unidade de Neurologia do Hospital de Base do Distrito Federal, está de acordo com a realização, neste setor, da pesquisa "ACHADOS ELETROENCEFALOGRÁFICOS EM PACIENTES PORTADORES DE ACIDENTE VASCULAR CEREBRAL", de responsabilidade do pesquisador Pierre da Silva Paz Junior, para estudo da relação entre acidente vascular cerebral e alterações eletroencefalográficas, após aprovação pelo Comitê de Ética e Pesquisa da Secretaria de Estado de Saúde do Distrito Federal - CEP - SES/DF.

O estudo envolve a realização de entrevistas, exames clínicos, realização de exame neurofisiológico (eletroencefalograma digital) em pacientes da SES-DF atendidos no HBDF. Tem duração total de 11 meses, com previsão de início para o mês de agosto/2014 e com coleta de dados com duração de aproximadamente 6 meses.

Brasília, 16 de julho de 2014.

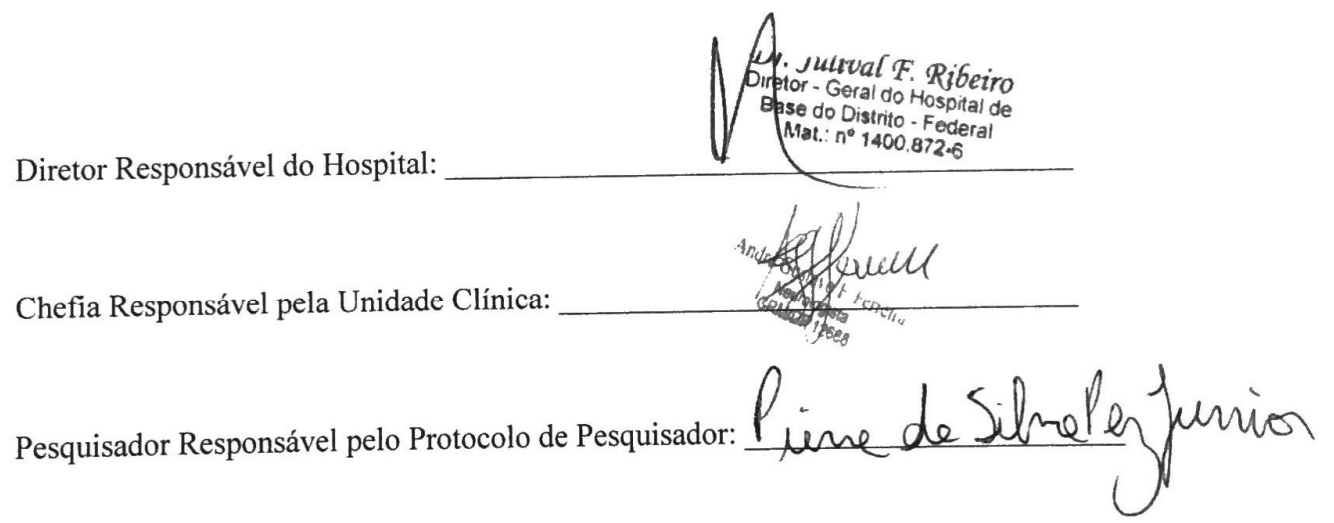




\section{ANEXO B ñ DOCUMENTAÇÃO FEPECS/SES/DF}

\begin{tabular}{|c|c|c|} 
SECRETARIA DE ESTADO DE \\
SAÚDE DO DISTRITO \\
FEDERAL - FEPECS/ SES/ DF
\end{tabular}

DADOS DO PROJETO DE PESQUISA

Título da Pesquisa: ACHADOS ELETROENCEFALOGRÁFICOS EM PACIENTES PORTADORES DE ACIDENTES VASCULARES CEREBRAIS EM UM HOSPITAL TERCIÁRIO DO BRASIL

Pesquisador: Pierre Paz

Versão: 2

CAAE: 33843314.2 .0000 .5553

Instituição Proponente:

DISTRITO FEDERAL SECRETARIA DE SAUDE

DADOS DO COMPROVANTE

Número do Comprovante: $\quad$ 061296/2014

Patrocionador Principal: $\quad$ Financiamento Próprio

Informamos que o projeto ACHADOS ELETROENCEFALOGRÁFICOS EM PACIENTES PORTADORES DE ACIDENTES VASCULARES CEREBRAIS EM UM HOSPITAL TERCIÁRIO DO BRASIL que tem como pesquisador responsável Pierre Paz, foi recebido para análise ética no CEP Secretaria de Estado de Saúde do Distrito Federal - FEPECS/ SES/ DF em 23/07/2014 às 09:21.

Endereço: SMHN 2 Qd 501 BLOCO A - FEPECS 